\title{
Video Article \\ Abbiategrasso Brain Bank Protocol for Collecting, Processing and Characterizing Aging Brains
}

\author{
Tino Emanuele Poloni ${ }^{* 1,4}$, Valentina Medici ${ }^{*}$, Arenn Faye Carlos ${ }^{1}$, Annalisa Davin ${ }^{2}$, Arcangelo Ceretti ${ }^{1}$, Michela Mangieri ${ }^{1}$, Paola Cassini $^{1,4}$, \\ Roberta Vaccaro ${ }^{3}$, Daniele Zaccaria ${ }^{3}$, Simona Abbondanza ${ }^{3}$, Matteo Bordoni ${ }^{5}$, Valentina Fantini ${ }^{2,6}$, Elena Fogato ${ }^{7}$, Cristina Cereda ${ }^{5}$, Mauro Ceroni ${ }^{8}$, \\ Antonio Guaita ${ }^{1,2,3}$ \\ ${ }^{1}$ Department of Neurology and Neuropathology, Golgi-Cenci Foundation \\ ${ }^{2}$ Laboratory of Neurobiology and Neurogenetic, Golgi-Cenci Foundation \\ ${ }^{3}$ Department of Neuropsychology and Social Sciences, Golgi-Cenci Foundation \\ ${ }^{4}$ Department of Rehabilitation, ASP Golgi-Redaelli Geriatric Hospital \\ ${ }^{5}$ Genomic and Post-Genomic Center, IRCCS Mondino Foundation \\ ${ }^{6}$ Department of Brain and Behavioral Sciences, University of Pavia \\ ${ }^{7}$ Department of Pathology, ASP Golgi-Redaelli Geriatric Hospital \\ ${ }^{8}$ Department of Neurological Science, IRCCS Mondino Foundation, University of Pavia \\ "These authors contributed equally
}

Correspondence to: Tino Emanuele Poloni at e.poloni@golgicenci.it, Arenn Faye Carlos at arennfaye.carlos@gmail.com

URL: https://www.jove.com/video/60296

DOI: doi:10.3791/60296

Keywords: Neuroscience, Issue 160, Brain Bank, neuropathology, aging, elderly care, longitudinal cohort study, brain donation, brain dissection protocol, QEEG, clinico-pathological correlation, omics

Date Published: 6/3/2020

Citation: Poloni, T.E., Medici, V., Carlos, A.F., Davin, A., Ceretti, A., Mangieri, M., Cassini, P., Vaccaro, R., Zaccaria, D., Abbondanza, S., Bordoni, M., Fantini, V., Fogato, E., Cereda, C., Ceroni, M., Guaita, A. Abbiategrasso Brain Bank Protocol for Collecting, Processing and Characterizing Aging Brains. J. Vis. Exp. (160), e60296, doi:10.3791/60296 (2020).

\section{Abstract}

In a constantly aging population, the prevalence of neurodegenerative disorders is expected to rise. Understanding disease mechanisms is the key to find preventive and curative measures. The most effective way to achieve this is through direct examination of diseased and healthy brain tissue. The authors present a protocol to obtain, process, characterize and store good quality brain tissue donated by individuals registered in an antemortem brain donation program. The donation program includes a face-to-face empathic approach to people, a collection of complementary clinical, biological, social and lifestyle information and serial multi-dimensional assessments over time to track individual trajectories of normal aging and cognitive decline. Since many neurological diseases are asymmetrical, our brain bank offers a unique protocol for slicing fresh specimens. Brain sections of both hemispheres are alternately frozen $\left(\right.$ at $\left.-80^{\circ} \mathrm{C}\right)$ or fixed in formalin; a fixed slice on one hemisphere corresponds to a frozen one on the other hemisphere. With this approach, a complete histological characterization of all frozen material can be obtained, and omics studies can be performed on histologically well-defined tissues from both hemispheres thus offering a more complete assessment of neurodegenerative disease mechanisms. Correct and definite diagnosis of these diseases can only be achieved by combining the clinical syndrome with the neuropathological evaluation, which often adds important etiological clues necessary to interpret the pathogenesis. This method can be time consuming, expensive and limited as it only covers a limited geographical area. Regardless of its limitations, the high degree of characterization it provides can be rewarding. Our ultimate goal is to establish the first Italian Brain Bank, all the while emphasizing the importance of neuropathologically verified epidemiological studies.

\section{Introduction}

According to $\mathrm{WHO}$, approximately 50 million people are currently suffering from dementia, a figure that is projected to triple by 2050 . Alzheimer's Disease is the major cause of dementia, followed by cerebrovascular disease and other age-related neurodegenerative disorders. In 2017, WHO developed the Global Dementia Observatory to raise awareness of dementia and encourage a global action plan against it ${ }^{1}$. Each individual has their own brain aging trajectory, therefore the search for the cure can be challenging due to the complexity of the pathogenesis of neurodegenerative diseases. Perhaps, each person possesses her or his own pathogenesis written in the brain tissue requiring a personalized approach. Thus, the study of brain tissue will be the key to understanding the mechanisms of neurodegeneration.

Looking back at the history of neuroscience, we realize that the most impressive and groundbreaking discoveries could never have occurred without the direct examination of the human brain. Throughout time, the source of brain tissue to be studied has changed from crude dissections, random 'chance encounters' and, in some cases, illegal trading, to organized brain collections and strategic modern brain banks. The consideration of many ethical aspects is one of the main factors differentiating modern brain banks from the brain collections of the past. The first real modern Brain Banks (BBs) were instituted in the second half of the $20^{\text {th }}$ century. Nicholas Corsellis and Wallace Tourtelotte can be considered pioneers of modern brain banking. In the UK, Corsellis assembled a collection holding over 1000 well-documented brains affected with various mental and neurological disorders ${ }^{2}$. Furthermore, Corsellis helped reveal the need to preserve fresh brain tissue in ice for the sake of biochemical testings ${ }^{3}$. Meanwhile in the USA, Wallace Tourtelotte introduced antemortem brain donation programs to facilitate solicitation of 
potential brain donors and ensure that the brains collected are accompanied by a complete medical and neurological history ${ }^{4,5}$. For a historical overview of brain collections and modern BBs, see Carlos et al. ${ }^{6}$.

So, why do we still need human brains? Brain diseases can only be given a definite diagnosis following neuropathological examination. Neuropathology challenges the clinical diagnosis, and is the key to a correct interpretation of the clinical symptoms and to the discovery of the histological bases of new syndromic variants. Indeed, the diagnosis can be redefined based on the pathological picture. Nonetheless, the autopsy rate has declined in the last decades due to recent development of innovative neuroimaging techniques. Through brain imaging, the morphological, functional and metabolic alterations in the brain, as well as the extent of protein misfolding, can be appraised in vivo. However, in vivo neuroimaging and other biomarker studies can only give an "estimate" of the pathological picture as they are unable to detect subtle cellular and molecular changes. Advances in molecular imaging and the discovery of new biomarkers, target molecules, and tracers ${ }^{7}$ (e.g., amyloid, TAU, microglial tracers) render the human brain even more indispensable to the interpretation of data obtained from clinical evaluations and biomarker testing. Furthermore, the omics technologies (genomics, epigenomics, transcriptomics, metabolomics, proteomics, lipidomics, etc.), performed on fresh and frozen brain tissue, opened up new possibilities for understanding disease mechanisms and discovering risk genes, novel diagnostic and prognostic markers, and potential drug targets $8,9,10,11,12,13,14,15,16,17,18,19,20,21,22,23$

For these purposes, modern BBs archive well-characterized, high-quality brain tissues making them available for the scientific community ${ }^{3,24}$. The brains supplied by BBs should be accompanied by a complete clinical history. The activity of a BB includes the following: (1) Recognition and recruitment of diseased and healthy individuals to brain donation programs; the ideal condition would be to achieve a multi-disciplinary follow-up of donors throughout life to obtain a complete clinical, lifestyle and social history, and biomarker profiles; indeed, cognitive reserve and brain structure depend on lifestyle and socio-educational factors ${ }^{25,26}$, so these information enriches the total data at hand. (2) Acquisition of the brain (consisting of cerebrum, cerebellum and brain stem) and related tissues (e.g., spinal cord, cranial nerves and ganglia, etc.) following the donor's demise, all the while observing standardized legal and ethical regulations. (3) Appropriate processing (dissection, fixation, freezing) of the brain, as defined in a standardized operative protocol, to obtain high quality tissue and to allow for future use in multidisciplinary research. (4) Detailed neuropathological characterization providing a final definite diagnosis. (5) Storage and distribution of tissue material to research community $^{27,28}$

All BBs store both frozen and formalin-fixed-paraffin embedded tissues. Each BB has its own protocol. Except for particular studies, such as the bihemispheric cutting protocol of the Biomedical Research Institute (New Jersey) ${ }^{29}$ and the Deramecourt's study of cerebrovascular pathology ${ }^{30}$, the biggest BBs in the world simply cut the cerebrum, cerebellum and brainstem along the midline (sagittal plane). One half is dissected fresh and then frozen for biochemical studies, while the other is fixed in formalin for histopathological assessment. So, biochemical and histopathological analyses are conducted separately on each hemisphere. The decision as to which side is fixed or frozen (laterality), depends on the singular bank ${ }^{31,32,33,34,35}$. As many neurological diseases are asymmetrical, our BB offers a unique protocol for slicing fresh brains: adjacent sections of the brainstem and each hemisphere are alternately fixed and frozen; a fixed slice on one hemisphere corresponds to a frozen one on the other hemisphere. Through this method, the use of brain tissue is optimized, and a complete histological characterization of all frozen material can be achieved with the possibility to obtain and compare histological and biochemical information from all areas of both hemispheres.

The frame of our brain bank project is the town of Abbiategrasso. Abbiategrasso is a small town about $22 \mathrm{~km}$ southwest of the city of Milan, in Northern Italy. It has a population of about 32,600 people. It is home to the Golgi-Cenci (GC) Foundation. GC Foundation is part of a large Rehabilitation Geriatric Hospital (ASP Golgi-Redaelli), and is an institute focusing on research about aging and care of the elderly. Particularly, it focuses on studying mental aging, the social and behavioral factors influencing it, and the biology and pathology underlying age-dependent neurocognitive disorders (NCDs). In 2009, the GC Foundation launched a new longitudinal study with 1321 participants (out of 1644 eligible subjects: initial response rate of 80.3\%) born between 1935 and 1939 (aged between 70-75 years), of Caucasian ethnicity, living in the same small geographic area. The study was called InveCe.Ab (Invecchiamento Cerebrale Abbiategrasso; in English: Brain Aging Abbiategrasso, ClinicalTrials.gov, NCT01345110) and is currently ongoing. InveCe.Ab is planned to obtain a cohort with maximum homogeneity and least variability in order to assess the incidence, prevalence and natural history of dementia, along with its possible risk or protective factors, including behavioral, psychosocial, clinical and biological variables ${ }^{36}$. The cohort's characteristics are shown in Figure 1 and Table 1 . The epidemiological data are consistent with the dementia trend in European population ${ }^{37,38}$ and InveCe.Ab participants have homogeneous genetic and environmental characteristics, representing a good model to study the trajectory from normal aging to neurocognitive disorders. Indeed, homogeneous populations require fewer subjects to reach adequate statistical power. The InveCe.Ab methodology has already been reported elsewhere ${ }^{36}$ but it is important to underline its multi-dimensional approach through periodic checks (every 2-3 years) using the same set of evaluations including: blood sampling (metabolic panel, homocysteine and vitamins, DNA extraction to profile Apolipoprotein E (APOE) and other genetic polymorphisms related to cognition and aging), anthropometric measurements (weight, height and waist), Talking While Walking Test (dual task test), an interview to appraise lifestyle (Mediterranean diet adherence, levels of physical activity and cognitive engagement) and social factors (social involvement, loneliness), a neuropsychological evaluation and a clinical general examination. To compare such longitudinal data with postmortem neuropathological data would be crucial for research. Therefore, our team and particularly Dr. Michela Mangieri conceived the neuropathological approach mentioned above. Since 2014 and during the second follow-up, the InveCe.Ab participants were asked to donate their brains, and thus bringing about the birth of the Abbiategrasso Brain Bank (ABB). The core donors of $A B B$ are the InveCe.Ab participants but ABB is now open to other volunteer donors. They are patients from ASP Golgi-Redaelli, home to several patients afflicted with different neurological diseases or adult volunteers who learn about the ABB Project and belong to the same geographic area (Abbiategrasso and surroundings). All donors undergo the same evaluation protocol.

The authors propose a method to track individual trajectories of normal aging and the possible progression to NCDs, and to accurately manage, process and characterize brains acquired from such donors followed longitudinally. Moreover, our aim is to meet and engage individuals in periodic neurological assessments, seminars and educational activities regarding the well-being of the brain and to raise their awareness of brain donation for research purposes. 


\section{Protocol}

In line with our institution's Human Research Ethics Committee and the BNE Code of Conduct, the ABB performs its activities following ethical standards $^{39,40}$. The brain harvesting procedure was submitted to and approved by the Ethics Committee of the University of Pavia in the context of the InveCe.Ab study ${ }^{36}$. The study procedures were in accordance with the principles outlined in the Declaration of Helsinki of 1964 and the following amendments. The consent form is complete and easily comprehensible. Joining the donation program is a personal decision, and complete awareness is needed. In case a person is not deemed competent to sign the consent form, authorization from legal guardian or nextof-kin (NOK) is warranted. Table 2 reports inclusion and exclusion criteria for brain donation. The research was performed under the supervision of the Federazione Alzheimer Italia.

\section{Recruitment to the brain donation program}

1. Convene the subjects who had expressed interest in brain donation and their NOK and/or legal guardian to present the project (the scope of the donation program; the process of brain removal, conservation, use and distribution), the right to withdraw from the program at any given time, the protection of anonymity, and the follow-up strategies. Discuss the possibility of additional biomarker investigations and genetic tests. Note the wishes of the potential donor or his NOK to be informed about the results.

2. Explain all the economic aspects including the lack of financial gain for both the Foundation and the donor (brains are donated and distributed altruistically). Inform them that ABB covers the cost of body transportation, while the family covers those of the funeral, burial or cremation.

3. In case of acceptance, obtain the signature of the consent to participate in the donation program. Give the donor an individual numerical code to guarantee anonymity. Write down the identification number of those participating in the longitudinal study and provide another code for the brain bank to easily separate the two subgroups of donors (participants or non-participants in the longitudinal study; see Table 3).

4. Give the donor an identification card that declares his status as a donor and displays the telephone number (available 24/7) to notify the Brain Bank of his death.

\section{Donor evaluation and serial follow-ups}

NOTE: It is possible to give consent only for some, and not all, of the below mentioned examinations. All clinical assessments are performed by the same team including a neurologist, a geriatrician with expertise in neurology and 3 psychologists. If new symptoms develop or the cognitive decline progresses, the time interval between follow-ups can be shortened.

1. As in the case of the InveCe.Ab study, obtain a lifestyle-social questionnaire, including degree of autonomy (ADL, IADL), personal habits, social and lifestyle factors that may affect mental functions. Collect family, medical and neurological history with information regarding genetic, infective, toxic, metabolic, autoimmune, cardiovascular, traumatic, degenerative, neoplastic, and neurological diseases. Collect previous clinical exams and list pharmacological treatments.

2. Carry out an extensive neuro-motor evaluation including testing for cranial nerves function, fundus oculi, visual field, muscle strength and tone, sensibilities, tendon reflexes, exteroceptive and primitive reflexes, meningeal signs, posture, gait, movements, coordination, sphincteric functions, and any presence of focal or Babinski signs. Evaluate language, mental status and any change in behavior.

3. Carry out an extensive neuro-cognitive evaluation including global cognition and a complete neuropsychological assessment of specific cognitive domains: verbal and visual memory, attention, psychomotor speed, language, semantic memory, executive functions, and visuospatial abilities (Table 4 for details). Consider the presence of depression (CES-D scale).

4. Obtain blood sample which is used for both blood chemistry analysis (Table 5) and isolation and storage of DNA, plasma and peripheral blood mononuclear cells (PBMC). Determine APOE genotyping (rs429358 and rs7412) (a more extensive genetic profiling has been determined in InveCe.Ab participants; see Table 6). Register an ECG (cardiac alterations, atrial fibrillation) and a resting state Electroencephalogram for Quantitative analysis (QEEG).

5. Consider optional biomarker testing including cerebrospinal fluid (CSF) TAU, phospho-TAU and $\beta$-amyloid, and brain imaging (MRI to detect in vivo degeneration, ischemia or inflammation; FDG-PET to detect metabolic failure; PIB-PET to detect brain amyloidosis related to Alzheimer's pathophysiology).

6. Plan the brain donor's subsequent check-up program. Set up the time intervals according to different age groups (up to 64 years old: every 10 years, 65-74 years old: every 3 years, from 75 years onwards: every 2 years).

7. Assign a clinical diagnosis and/or a neurocognitive diagnosis according to DSM-V. Classify clinical dementia staging with Clinical Dementia Rating (CDR) score. Update CDR in the last period before death.

8. Prepare a database graphically similar to the paper one. Insert all collected data into the Brain Bank database. Plan a revision by another clerk to check for any mistakes.

\section{Time of death and brain removal}

NOTE: The Italian law establishes that asystole must last longer than $20 \mathrm{~min}$ to confirm death. The registration of a flat electrocardiogram (ECG) for at least $20 \mathrm{~min}$ (named thanatography) allows for an autopsy to be carried out within $24 \mathrm{~h}$ of death (DPR 285/90 art. 8 and Law n. 578 Dec 29,1993 ). A postmortem time of $<24 \mathrm{~h}$ is a good target to preserve the overall tissue quality. In case of a postmortem time $>30 \mathrm{~h}$ the autopsy is cancelled (see Table 2). The autopsy team is composed of a pathologist, a neurologist and/or a neurobiologist, a nurse, an anatomical room technician and any trainee students; the first two team members also perform the neuropathological diagnosis. During cadaver handling and dissection, the use of appropriate clothing (coat, gloves, eyeglasses and hairnet) is mandatory. In this section, we describe the main tools and equipment normally used in our laboratory. Readers can choose the instruments to be used at their discretion. For a detailed description of the materials herein utilized, please see Table of Materials. 
1. Make sure that the funeral agency chosen by the family brings the body to the ABB facilities. Perform the thanatography during which the cardiac activity must be absent. If so, sign a death certificate and begin the autopsy procedures.

2. Transport the cadaver to the autopsy room. Measure the circumference of the skull at the level of the widest part of the head. Measure from the nasion to the inion to obtain the anteroposterior diameter (APD), while the distance from one ear to the other yields the transverse diameter (TD). Calculate the cephalic index $(\mathrm{Cl})$ using the formula: $\mathrm{Cl}=$ TD/APD $\times 100$.

3. Using a sharp scalpel, make a scalp incision on the coronal plane, from the tip of the mastoid process on one side to the other, passing over the vertex. Cut through hair, skin and subcutaneous tissue and separate the two folds of the scalp carefully from the underneath skull. Perform the incision until the yellow supraorbital fat becomes visible and reflect the scalp anteriorly. Pull the other part posteriorly.

4. Using scalpel and forceps, take a small sample of the temporal muscle at each side, put one in $4 \%$ formaldehyde and freeze the other (see section 7).

5. Cut the skull using an electrical saw following a V-cut on the frontal side. Remove the skullcap and cut the meninges. Sample pieces of dura are both fixed in $4 \%$ formaldehyde and frozen (see section 7 ).

6. Obtain about $10 \mathrm{~mL}$ of CSF by inserting a $20 \mathrm{G} 3.5 \mathrm{in}$. needle through the corpus callosum to reach the third ventricle. Assess the appearance, color and turbidity of $\mathrm{CSF}$ and measure the $\mathrm{pH}$. Then, make 10 aliquots of about $1 \mathrm{~mL}$ each and store them at $-80^{\circ} \mathrm{C}$.

7. Lift up the brain delicately pulling on both frontal lobes and cut both optic nerves infundibulum, the internal carotid arteries (ICA) and the third fourth, fifth and sixth cranial nerves. Cut the tentorium to reach the posterior fossa and cut vertebral arteries and lower cranial nerves. Insert the scalpel as deep as possible through the foramen magnum, to cut the most caudal portion of the medulla. At this point, remove the whole brain gently.

8. With the scalpel, pierce the bone over Meckel's cave and obtain the Gasserian ganglion from both sides. Fix one ganglion in $4 \%$ formaldehyde and freeze the other (see section 7). Fracture the bony sella turcica using a surgical mallet and chisel to remove the pituitary gland and then fix it in $4 \%$ formaldehyde.

9. Inspect the skull and the whole brain for macroscopic alterations and vascular changes. With a measuring tape, measure the brain obtaining the transverse and anteroposterior diameters. Retrieve with care the circle of Willis and assess it macroscopically (Figure $2 \mathrm{E}$ ) for the presence of anatomic variants, lesions or vessel stenosis.

10. Take 1-2 pieces of leptomeninges of $2-4 \mathrm{~cm}^{2}$ from the convexity and preserve them in complete high glucose Dulbecco's modified Eagle media (DMEM) culture medium at $4{ }^{\circ} \mathrm{C}$ containing $20 \%$ fetal bovine serum (FBS), $1 \%$ pen/strep, $1 \%$ glutamine and $1 \%$ non-essential aminoacids (as previously published, with some modifications) ${ }^{41}$.

1. In order to obtain fibroblasts cultures, place the leptomeninges on a $10 \mathrm{~cm}$ Petri dish and wash twice with phosphate buffer saline (PBS), each time discarding the liquid.

2. Using a scalpel and a pipette tip, cut the leptomeninges into small pieces of about 2 or $3 \mathrm{~mm}$, seed 3-4 pieces in each well of a 6-well plate previously coated with gelatin at $0.5 \%$ and fill each well with $2 \mathrm{~mL}$ of complete medium supplemented with $1 \%$ of amphotericin $B$ (conduct the processing in a biosafety cabinet to guarantee the sterility of the culture).

3. Place the plate into a $37{ }^{\circ} \mathrm{C}, 5 \% \mathrm{CO}_{2}$ incubator for at least one week and change spent medium every $3-4$ days. Trypsinize cells with sterile $1 \mathrm{x}$ trypsin when the well is at about $70 \%$ of confluence. Place leptomeningeal fibroblasts into a T75 flask and fill it with $12 \mathrm{~mL}$ of complete medium. After 5 days trypsinize and preserve them in $900 \mu \mathrm{L}$ of FBS and $100 \mu \mathrm{L}$ of dimethyl sulfoxide (DMSO).

11. Separate and inspect cerebrum, cerebellum and brainstem and weight them individually. Take off the pineal gland and fix it in $4 \%$ formaldehyde. Remove olfactory bulbs and optic nerves, and fix or freeze one of each pair, independently of the side. Keep the cerebrum, cerebellum and brainstem in ice at $4{ }^{\circ} \mathrm{C}$ for at least $2-4 \mathrm{~h}$ until ready for dissection to minimize tissue disruption and reduce the softness of tissue.

12. After the harvesting, pay appropriate attention and care to the cadaver. Reattach the bone using a super adhesive glue, and stitch back the scalp using a surgical needle and non-absorbable sutures.

13. Show respect and gratitude to the deceased person by treating the cadaver gently. Clean and shave the face, and wash and dry the hair, because the cadaver will be put in an open coffin visible to loved ones.

NOTE: Recomposition of the cadaver is done by a technician. The protocol can be paused here.

\section{ABB dissection protocol}

NOTE: The same pathologist and/or neurologist slice the brainstem, cerebellum and cerebrum under a fume hood. A neurobiologist arranges the slices after sectioning. A trainee not handling brain sections documents the whole procedure with photos to be uploaded to the database to serve as guide during the subsequent tissue processing phases.

1. Using a dissecting knife, cut the brainstem axially and make the first cut at the level of rostral midbrain, through the superior colliculus, to obtain two slices exposing the substantia nigra (SN).

2. Make the rest of the cuts to acquire $8 \mathrm{~mm}$ brainstem slices to obtain about 10 sections. Be careful to include cuts passing through the rostral pons near the superior margins of the fourth ventricle to observe the locus coeruleus (LC) and through the medulla oblongata inferior to the acoustic striae just above the inferior apex of the fourth ventricle to have slices including the dorsal motor nucleus of the vagus (DMNV).

3. Name all slices in a rostrocaudal sense as BS (brainstem) followed by Arabic numbers to identify the sections. After the last brainstem section, use the designation SC (spinal cord) followed by numbers $1-n$. About $2-4$ SC slices are obtained depending on the number of spinal metameres removed (Figure $\mathbf{2 H}-\mathrm{I}$ ).

4. Label all sections to be fixed or to be frozen alternately and take a picture for the photo archive (Figure 2). Then, fix and freeze all slices as described below (steps 4.7 and 4.8).

5. Cut the cerebellum on the sagittal plane to separate the two cerebellar hemispheres at the level of the vermis. Perform sagittal sectioning to obtain 5 slices from each hemisphere (Figure 2F-G).

6. Name all slices from vermis as CBR or CBL (for right and left cerebellum respectively) and use Arabic numbers to identify the sections. Label all sections to be fixed or frozen alternately and take a photo for the archive (Figure 2). Then, fix and freeze all slices as described below.

7. Separate the two hemispheres of the cerebrum through the corpus callosum (Figure 2A-B) and slice them singularly on the coronal plane. Make the first cut in a plane passing between the optic chiasm and the mammillary bodies, through frontal lobe, temporal pole, anterior cingulate, anterior commissure, nucleus of Meynert and basal ganglia. 
8. Perform the second cut about $1 \mathrm{~cm}$ posteriorly passing through the mammillary bodies and exposing the basal ganglia, anterior thalamus, subthalamus and amygdala. Continue slicing to dissect the anterior and posterior regions to acquire $1 \mathrm{~cm}$ slices in order to obtain 15 to 20 sections for each hemisphere.

9. Set the slices on a flat surface. Lay them following their original position in the anteroposterior direction, from the frontal to the occipital pole, with the portion continuous with the previous slice facing upwards.

10. By comparing the sections from both hemispheres, select alternate sections from each hemisphere to be retained as fixed or frozen material. Recognize the main sections to be fixed and processed for histopathology including frontal and temporal lobes, cingulate, basal ganglia, nucleus basalis of Meynert, amygdala, thalamus, hippocampus and entorhinal cortex, occipito-temporal gyrus, parietal and occipital lobes

11. Name all slices in anteroposterior sense as $L$ (left) or $R$ (right) followed by Arabic numbers and put a label indicating whether the slice is to be fixed or frozen (Figure 2A-D). To identify the sections, take a picture for the photo archive. Then, fix and freeze all slices as described in sections 7 and 8 .

\section{Brain and vessels inspection and macroscopic pathological assessment (with the naked eye)}

1. Carry out a general macroscopic examination considering meningeal alterations, diffuse or localized cortical atrophy, hippocampal atrophy, ventricular enlargement, cerebellar atrophy, brainstem atrophy, substantia nigra pallor, white matter alterations (specify type and location).

2. Examine the circle of Willis considering atherosclerosis and occlusion. Assign score $=1$ in the presence of atheroma without stenosis of more than $50 \%$, score $=2$ if at least one artery is $50 \%$ or more occluded, score $=3$ if two or more arteries are $50 \%$ or more occluded ${ }^{42}$. Evaluate the presence or absence of pathology in other intracranial vessels.

3. To detect parenchymal vascular lesions, examine brain hemispheres, cerebellum and brainstem. Consider lacunar lesions (diameter $<10$ $\mathrm{mm}$ ), ischemic or hemorrhagic infarcts and hemorrhage (diameter $>10 \mathrm{~mm}$ ). If present, specify size in millimeter, number and location. Also, consider the presence or absence of subarachnoid hemorrhage.

\section{Tissue quality control}

NOTE: Agonal factor score (AFS) ranges between 0 and 2 and is important in the evaluation of tissue quality. To determine AFS, consider clinical conditions occurring around the time of death (particularly conditions determining brain acidosis) and the duration of agonal state (sudden death or prolonged agony). If AFS $>1$, the brain tissue might not be of optimal quality ${ }^{43}$. Also consider brain and $\mathbf{C S F} \mathbf{p H}$; if $\mathrm{pH}<6$, the brain tissue might not be of optimal quality ${ }^{43,44}$.

1. Verify if death has been caused by conditions that can damage brain tissue including hypoxia, protracted acidosis, mechanical ventilation, multi-organ failure, high fever, severe cranial trauma, ingestion of neurotoxic substances, severe dehydration, severe hypoglycemia, epileptic state and prolonged coma. If one of these conditions is present, assign 1 point.

2. Verify the duration of agonal state (rapid if death occurs within $1 \mathrm{~h}$, intermediate if death occurs between 1 and $24 \mathrm{~h}$, slow if the agonal state lasts more than 1 day). If an intermediate or a slow death happens, assign 1 point.

3. Calculate AFS score, and measure $\mathrm{CSF}$ pH by an electrode $\mathrm{pH}$ needle and tissue $\mathrm{pH}$ by a surface $\mathrm{pH}$ meter on a brain slice from each lobe and on a cerebellar section.

\section{Tissue freezing}

1. Keep all tissues to be frozen in ice at $4{ }^{\circ} \mathrm{C}$. Then, freeze them quickly. Place them on a prefrozen aluminum tray. Cover with an interlocking aluminum plate to keep them flat. Put them in liquid nitrogen at $-120^{\circ} \mathrm{C}$ for $3 \mathrm{~min}$.

2. Place the slices inside a plastic bag labeled with their corresponding identification code and slice number. Divide the plastic bags into three cryogenic boxes (for right hemisphere, left hemisphere and brainstem) and store at $-80^{\circ} \mathrm{C}$.

\section{Tissue fixation}

1. Wrap the slices in gauze one by one, and put the slices in $10 \%$ phosphate buffered formalin solution. After 1 day, substitute the formalin solution with a fresh solution. Leave them for about 5 days in a cold room at $4{ }^{\circ} \mathrm{C}$.

2. Keep all slices as a whole and split only the slices containing hippocampus and amygdala in two parts (Figure 3). The upper part of both slices will contain the frontal lobe (R10a-L9a in Figure 3); the lower parts will contain respectively: (1) amygdala, temporal lobe and basal ganglia ( $9 \mathrm{~b}$ in Figure 3B), and (2) hippocampus and part of temporal lobe (R10b in Figure 3A). This is made in order to maintain the relationship between the various structures.

3. Put all sections in phosphate buffer for at least 2 days to wash out and remove cross linking products. NOTE: The protocol can be paused here.

\section{Dehydration, clearing, paraffin embedding and slide preparation}

1. Prepare increasing concentrations of ethyl alcohol from $70 \%$ to $100 \%$, xylene and two sets of molten paraffin wax. Place the brain sections in the automatic processor for dehydration, clearing procedure and paraffin infiltration (use two different tissue processing protocols for macro (cerebrum and cerebellum slices) and micro samples (brainstem slices and the other small samples); Table 7). Embed the tissue in paraffin wax on a metal or plastic mold.

2. Select the sections to slice for evaluating all the regions of interest applying Montine's index for neuropathological characterization ${ }^{45}$ (Table 8). Then, slice the selected sections. 
3. Use a sledge microtome for macrosections and a rotary microtome for small sections. Cut $5 \mu$ m slices for Haematoxylin and Eosin (H\&E) staining and $8 \mu \mathrm{m}$ slices for the other stainings and immunohistochemistry. Put the slices on three different kind of histological slides: $8.5 \mathrm{~cm}$ $\times 11 \mathrm{~cm}$ for biggest slices, $5 \mathrm{~cm} \times 7.5 \mathrm{~cm}$ for medium slices and the classic $2.5 \mathrm{~cm} \times 7.5 \mathrm{~cm}$ for smallest ones. NOTE: The protocol can be paused here.

\section{Deparaffinization, histological staining and immunohistochemistry (IHC)}

1. Perform deparaffinization to enable reaction with aqueous dye solutions. Perform it using xylene and decreasing alcohol concentration (5 min for every step). Rehydrate for 5 min with distilled water.

2. Use the following histological stainings to evaluate architectural and structural tissue abnormalities, and cellular morphology: H\&E (for vascular microscopic lesions and inflammation), Cresyl Violet (NISSL; for neuronal loss), Luxol Fast Blue (LFB; for demyelination), Gallyas (for neuritic plaques and neuropil threads).

3. Use immunohistochemistry (IHC) in order to highlight the presence of specific target structures. Anti-NeuN and anti-GFAP are used to identify neuronal and glial compartments; 4G8, AT8, $\alpha$-SYN and TDP-43 are used to identify proteins that aggregate in neurodegenerative diseases

(Table of Materials).

1. Pretreat dewaxed sections with $3 \% \mathrm{H}_{2} \mathrm{O}_{2}$ for 10 min then rinse in PBS. Perform retrieval treatment with citrate buffer $0.01 \mathrm{M}$ pH 6 (microwaved serially for 2, 1 and $2 \mathrm{~min}$ ) for $4 \mathrm{G} 8$, a-SYN, TDP-43 and NeuN antigens; use $70 \%$ formic acid for $4 \mathrm{G} 8$ and $\alpha-S Y N$. Preincubate for 30 min in $5 \%$ normal goat serum.

2. Incubate overnight at $4{ }^{\circ} \mathrm{C}$ with the primary antibody. On the day after, rinse the sections in PBS before incubation with the secondary antibody (Envision+ System-HRP labeled Polymer) at a dilution of 1:2 in PBS for $1 \mathrm{~h}$ at room temperature.

3. Wash several times in PBS and incubate in chromogen system with diaminobenzidine (Liquid DAB+Substrate Chromogen System) looking at reaction development under the microscope (magnification 4-10x). Finally wash the sections in PBS. Counterstain the sections in haematoxylin, dehydrate and coverslip with DPX mounting.

NOTE: Table 8 summarizes the standard protocol. Perform H\&E staining on all sections, while special/specific stains and reactions are used on selected sections. Selected cases require additional areas or reactions. Table of Materials shows the details of antibodies used for IHC.

\section{Basic neuropathological characterization}

NOTE: Nonspecific brain tissue alterations, vascular pathology, Alzheimer's Disease (AD) pathology, non-AD TAUopathies, synucleinopathies, TAR DNA-binding Protein (TDP-43) pathology and hippocampal lesions are studied. An optical microscope connected to a camera is used to detect parenchymal microscopic lesions. The histological assessment is performed by the same team of trained personnel in neuropathology, including a professor of neurology, a neurologist and a pathologist. It is based on a modified Montine's approach ${ }^{45}$ (Table 8) also including: (1) Heterogeneous non-AD TAUopathies which constitute the pathologic hallmark of the Fronto-Temporal Lobe Degeneration (FTLD) related to TAU deposits: Pick's disease, non-fluent Primary Progressive Aphasia (TAU-nfPPA), Progressive Supranuclear Palsy (PSP) ${ }^{46,47}$ and CorticoBasal Degeneration $(C B D)^{48}$. In addition, non-AD TAUopathies include conditions related to aging and with no definite clinical significance such as Primary Age-Related TAUopathy (PART) ${ }^{49}$, Age-Related TAU Astro-Gliopathy (ARTAG) ${ }^{50}$, argyrophilic grain disease ${ }^{48}$. (2) Lewy Type Synucleinopathy (LTS) that is related to Parkinson's Disease (PD) and Lewy Bodies Dementia (LBD). To search for LTS, apply the following hierarchic steps: at first, olfactory bulb, brainstem, amygdala/temporal cortex; if the previous areas are positive, add limbic structures (hippocampal formation, entorhinal cortex, anterior cingulate), middle frontal gyrus, inferior parietal lobule and occipital cortex ${ }^{45}$. If clinical features of cortical LBD are present (i.e., fluctuations and/or hallucinations), consider limbic structures and isocortex for the first step. (3) TDP-43 deposits, the pathologic hallmark of FTLD related to TDP-43 deposits: behavioral variant of Fronto-Temporal Dementia (bvFTD), Semantic Dementia (SD or SVFTD), TDP-nfPPA and FTD-Motor Neuron Disease (FTD-MND). The IHC for TDP-43 is performed on the following sections: amygdala, hippocampus, entorhinal cortex and middle frontal gyrus; in cases with suspected clinical FTLD, consider to examine other sections ${ }^{51}$.

1. Evaluate nonspecific brain tissue alterations in selected sections using H\&E, NISSL, LFB, and IHC for GFAP and NeuN. Consider neuronal rarefaction, ballooned neurons, spongiosis, gliosis, myelin loss, the presence or absence of inflammatory or tumoral infiltrates. Specify the prevalent location of these alterations.

2. To detect microscopic vascular lesions, examine H\&E and LFB slides including at least two hemispheric macro-sections (the first passing through the mammillary bodies (Charcot cut) with frontal and temporal lobes, basal ganglia, anterior thalamus, amygdala, and the second passing through the occipital lobe), the "geniculate" section of the hippocampal formation, one cerebellar section, and all three principal sections of the brainstem (through substantia nigra, locus coeruleus and DMNV).

1. Detect small vessel disease including arteriolosclerosis, lipohyalinosis, perivascular space dilatation, white matter loss, leptomeningeal and/or parenchymal and/or capillary cerebral amyloid angiopathy. Specify grading $(0-3)$ and prevalent location ${ }^{42,52}$. Consider the presence or absence of hemosiderin leakage, microbleeds and microinfarcts.

2. Evaluate the contribution of vascular damage to cognitive impairment using hierarchic Deramecourt's scheme (vessel wall alterations-small vessel disease-macroscopic infarcts). For this evaluation consider a frontal and temporal lobe section, basal ganglia and hippocampus (score $0-20)^{30}$. Also, estimate the probability that cerebral vascular disease contributed to the cognitive impairment using VCING score (low-intermediate-high), obtained by considering hemispheric macroscopic infarcts and small vessel disease of the occipital lobe including moderate to severe arteriolosclerosis and amyloid angiopathy ${ }^{53}$.

3. Evaluate AD pathology using IHC (4G8) for amyloid spread. Define Thal's stages (1-5) ${ }^{54}$, aggregate Amyloid scoring $(0-3)^{45}$, and morphology per site (diffuse, focal, cored).

1. Evaluate AD Tau pathology using IHC (AT8) and describe the prevalent morphology per site (neurofibrillary tangles, neuropil threads, neuritic plaques). Define Braak's stage (I-VI +; positive sign indicates the presence of additional areas of hyperphosphorylated Tau pathology not following the Braak's hierarchy $)^{55}$ and aggregate scoring $(0-3)^{45}$. 
2. Evaluate neuritic plaques using Gallyas staining and CERAD score $(0-3)^{56}$. Then, define the probability of the neuropathological features corresponding to an AD clinical syndrome using the Amyloid-Braak-CERAD score (ABC score 0-3): none-low-intermediatehigh $^{45}$.

4. Use AT8 IHC to notice the presence or absence of non-AD TAU pathology specifying prevalent location and peculiar morphologic picture. Consider neuronal inclusions such as flame shaped or globose tangles, spherical-globular inclusions (i.e., Pick's bodies) ${ }^{57}$, neuropil threads, dystrophic neurites, argyrophilic grains; and glial inclusions such as tufted astrocytes, thorny astrocytes, astrocytic plaques, coiled bodies, globular inclusions ${ }^{58,59}$.

5. Use $\alpha$-syn IHC to detect LTS (Lewy bodies, pale bodies and Lewy neurites) on the areas in the note above. In case of positivity, apply McKeith's grading (0-4) to evaluate the severity of the lesions ${ }^{60}$; then, use Beach's stages (I-IV) for topographical distribution (olfactory bulb, brainstem predominant, limbic predominant, neocortical) ${ }^{61}$. Compare Beach's and Braak's stages to define the relationship between LBD and AD pathology ${ }^{60,61,62}$

6. Consider the presence or absence of Glial Cytoplasmic Inclusions ( $\mathrm{GCl}$; non Lewy-type glial synucleinopathy) which are the pathological hallmark of Multiple System Atrophy (MSA) and specify their prevalent location ${ }^{63}$.

7. Use TDP-43 IHC to detect TDP-43 deposits on the areas in the note above. Identify the prevalent morphology per site: Neuronal Cytoplasmic Inclusions (NCls), Distrophic Neurites inclusions (DNs), Nuclear Inclusions (NIIs). Define the pattern: A (predominant NCls and DNs: bvFTD and nfPPA); B (predominant NCls: FTD-MND); C (predominant long DNs: svPPA and bvFTD) ${ }^{51,64}$. Use Nelson's scheme to define the presence of TDP-43 in AD cases ${ }^{65,66}$.

8. Evaluate the hippocampus starting from subiculum and Sommer sector (CA1). Use the Rauramaa's score (0-4): 1-2 for microinfarcts and macroinfarcts, respectively; 3-4 corresponding to moderate and severe neuronal loss, respectively, and hippocampal atrophy (hippocampal sclerosis: HS). Point out the presence or absence of pathological protein deposits (in decreasing order of frequency: pTAU, TDP-43, $\alpha-s y n)^{67}$

9. Define the neuropathological diagnosis and enter all the pathological data in the database.

NOTE: The rooms in which the biological material and sensible data of the donors are stored are always locked and only the authorized personnel are allowed to enter, in order to guarantee both security and privacy. The frozen biological material is stored in locked freezers at $-80^{\circ} \mathrm{C}$, while formalin-fixed paraffin-embedded tissues are stored in locked closets at room temperature. Dual motor freezers are used and a back-up freezer is also present. Furthermore, to ensure the freezers are always working, they are provided with a 24/7 monitoring system that sets off an alarm. An emergency generator is also present, in case of power failure. The participants are anonymized with a numerical code to ensure their privacy; it is not possible for non-authorized personnel to go back to the identity of the donors and their sensible data. All information is collected in a password-protected database; likewise, a hard copy of these data is kept in locked archives.

\section{Representative Results}

\section{Brain donors and brain harvesting data}

In 2014, donor recruitment began during the second InveCe.Ab follow-up, involving 1010 out of 1061 eligible subjects (response rate: $93 \%$; Figure 1). With relation to the InveCe.Ab longitudinal study, 290 out of 1010 participants $(28.7 \%)$ agreed to register as donors (160 already registered and 130 who expressed their intention to register). The educational level is higher in donors than in non-donors (66\% of our donors have a medium-high level of education: 8 or more years of school), indicating the importance of culture and education. Many "healthy" individuals also showed interest in the brain donation program. Most of our "controls" confess that they can sympathize with the diseased and their relatives, and want to contribute somehow to research leading to a better understanding of neurological diseases. Selfless people who regularly engage in blood or marrow donation programs during life are more open to the idea of brain donation, as are people who have already agreed to donate organs after death. They are aware of the fact that even though there would not be a living recipient, their donation would be of great importance to research. Another factor contributing to brain donation is the preference to be cremated. Currently, the ABB donor population includes a total of 427 individuals (290 InveCe.Ab participants +137 volunteers or patients from the ASP Golgi-Redaelli Geriatric Hospital), $75 \%$ of which are 70 years old or older. There is a clear predominance of females $(64 \%)$ and mentally intact elderly (about $85 \%$ ). So far, 27 brains have been harvested out of 40 deceased donors ( $67 \%$ autopsy rate); 13 subjects have not been autopsied for various reasons including failure to report death to $A B B$, severe injuries leading to brain destruction, dangerous infective diseases and 1 CJD case; 4 subjects have revoked their consent. Up till now, 24 out of the 27 brains harvested received a complete neuropathological characterization with a definite clinico-pathological diagnosis, while the remaining 3 brains are still under examination (Table 3). Additional brain harvesting data from ABB include: mean age at death (81 years); mean postmortem interval (10.37 h); mean CSF pH (6.64); mean tissue pH (6.07); average brain weight (1012.86 gr); AFS was 1 in $90 \%$ of deceased subjects.

\section{Neurophysiological biomarkers (QEEG)}

QEEG is part of the multi-dimensional approach. It is a straightforward, non-invasive and inexpensive method, with a likely potential to detect conversion from mild Neuro-Cognitive Disorder (mild-NCD or MCI) to major Neuro-Cognitive Disorder (major-NCD or dementia). All through our multi-dimensional approach, a plain QEEG examination is performed and its possible role as a biomarker for dementia is tested. Our data on a preliminary series of 36 brain donors (18 normal elderly (NOLD), 11 mild-NCD and 7 major-NCD; 9 of which with a definite neuropathological diagnosis) indicate that the mean alpha rhythm percentage was significantly lower in major-NCD compared to mild-NCD ( $p: 0,002)$ and NOLD (p: 0,033). Conversely, slower EEG frequencies (theta/delta) were significantly higher in major-NCD than in NOLD/mild-NCD (see the example cases in Figure 4). In our series, EEG rhythm distribution can differentiate NOLD/mild-NCD subjects from major-NDC patients regardless of the etiological diagnosis, suggesting that brain rhythms are influenced by the burden of degenerative lesions regardless of lesion type. Indeed, 7 out of 9 examined brains show dementia due to mixed pathologies. The specificity concerning the nature of the pathology would seem to be low and brain electrical rhythms appear to be influenced more by the burden and topography of the lesions than by their molecular nature (Poloni, et al. proceedings of AD/PD 2019, Lisbon, data unpublished).

\section{Emblematic cases}

The ABB protocol may be useful and necessary in some cases and conditions. An example is the presence of an asymmetric pathology (Figure 5). Our protocol is very suitable for the identification and proper characterization of this type of pathology. So far, we have examined 4 brains with asymmetric involvement, some of which are shown in Figure 5. Macroscopically, right side atrophy (Figure 5A) is present in one case with severe ventricular dilatation in the right coronal section (Figure 5C) compared to the left side (Figure 5B). Another case displays infarct of the 
right hemisphere (Figure 5D) and another one shows a clear atrophy of the right mammillary body (Figure 5E). At the microscopic level, a case of FTLD shows an asymmetrical TDP-43 positivity that is more intense in the right frontal side compared to the left (Figure 5F,G).

Macrosections (Figure 6A,C) are used to get an overall view. LFB staining helps to identify myelin loss (Figure 6D) and IHC for both 4G8 and AT8 (Figure 6B) allows to evaluate the distribution of the immunoreactivity in the hemispheres with the naked eye. In the ABB series, brains of related individuals are available to be compared.

In Figure 7, microscopic images of sections from the brains of homozygous twins are placed side by side for easier comparison (twin 1 (BB137): Figure 7A,C,E,G,I,K versus twin 2 (BB138: Figure 7B,D,F,H,J,L). As in a similar previous study ${ }^{68}$, both twins were compared by clinical and neuropathological assessments. The twins reported the same diagnosis of major-NCD due to multiple etiologies, but they died two years apart, at age 83 (dementia-onset at 72 years) and 85 (dementia-onset at 76 years), respectively. Their brains have a very similar neuropathological picture, with high AD pathology associated with amyloid angiopathy. 4G8 immunoreactivity is diffused throughout the cortex (Figure 7A,B) and basal ganglia (Figure 7C,D) with diffuse, dense, and cored plaques. It is also clearly detected in both parenchymal and leptomeningeal vessels of the cortex and the cerebellum (Figure 7A,B,G-J). At higher magnification, capCAA is clearly evident (Figure 7G,H). AT8 immunopositive plaques, tangles and threads are diffuse in the parietal cortices of both brains (Figure 7E,F,L). Regarding the amyloid and NFT pathology burden, twin 1 is considered a Thal stage 3 (montine A2) and a Braak stage 5 (montine B3), while twin 2 is considered a Thal stage 5 (montine A3) and a Braak stage 6 (montine B3). They had the same years of education and similar lifestyle; however, one (BB137) was married and became widowed soon after while the other was single (BB138). They showed different degrees of clinico-pathologic variability with the same beginning and same course of illness but in different time frames. This confirms the fact that, although the genetic component plays a key role in the development of the disease, the epigenetic and environmental component are fundamental in determining slightly different manifestations.

In some cases, the clinical picture does not correspond to the neuropathological features. In Figure 8, two different cases were clinically defined as AD cases; neuropathological analyses show, in addition to an intermediate AD pathology, a diffuse positivity for $\alpha$-syn. In the first case, a severe LTS (Beach IV) picture shows Lewy bodies homogeneously found throughout the gyrus cingoli (Figure 8A) and in SN as cytoplasmic inclusions surrounded by neuromelanin (Figure 8B,C). In the second case, Lewy bodies associated with Lewy neurites are diffusely distributed in the amygdala (Figure 8D,E) and in Meynert's nucleus (Figure 8F,G) suggesting a limbic LTS diagnosis. Indeed, the topography of lesions rather than their molecular nature produces the clinical manifestations.

\section{InveCe.Ab Study participants- flow chart 2010 - 2018 (4 waves)}

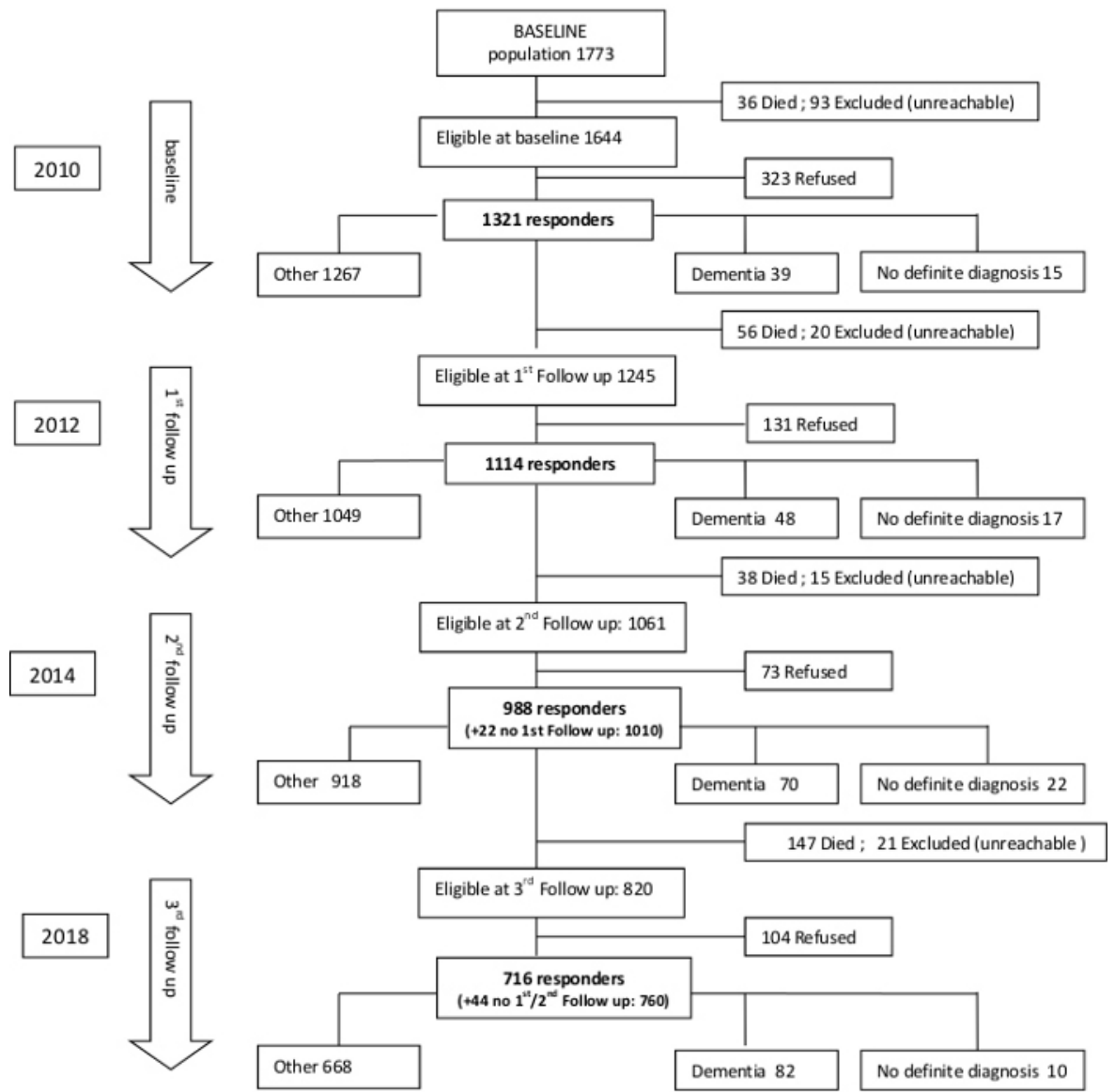

Figure 1: Flow chart of the Inve.Ce.Ab study. At baseline, the overall prevalence of dementia was $3 \%$. During the course of the follow-ups, the prevalence rates were: $4.4 \%\left(1^{\text {st }}\right)^{69}, 7.1 \%\left(2^{\text {nd }}\right), 10.9 \%\left(3^{\text {rd }}\right)$. The eight-year incidence rate was $15 \mathrm{p} / 1000 /$ year $(95 \% \mathrm{Cl}: 13-18 \mathrm{p} / 1000 / \mathrm{year})$. Donor recruitment started in 2014 (during the second follow-up). Please click here to view a larger version of this figure. 

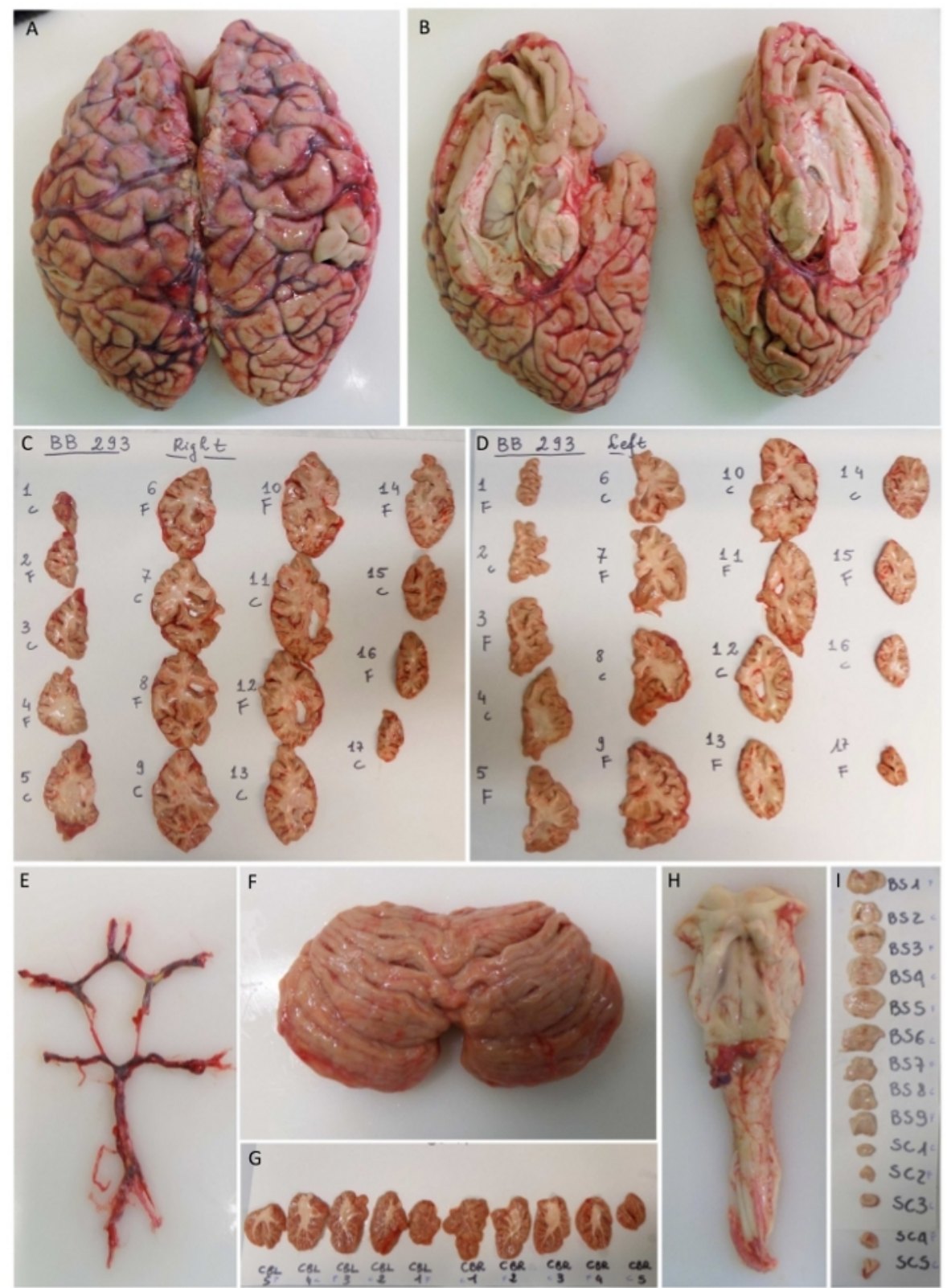

Figure 2: Dissection protocol of cerebrum (A), cerebellum $(\mathbf{F})$ and brainstem $(\mathbf{H})$. Circle of Willis and single hemispheres are shown in (E) and (B). Coronal cuts of right (C) and left side (D) are numbered and alternatively fixed ("F") and frozen ("C"). Sagittal cerebellum sections (G) and brainstem axial sections (I) are shown. Please click here to view a larger version of this figure. 

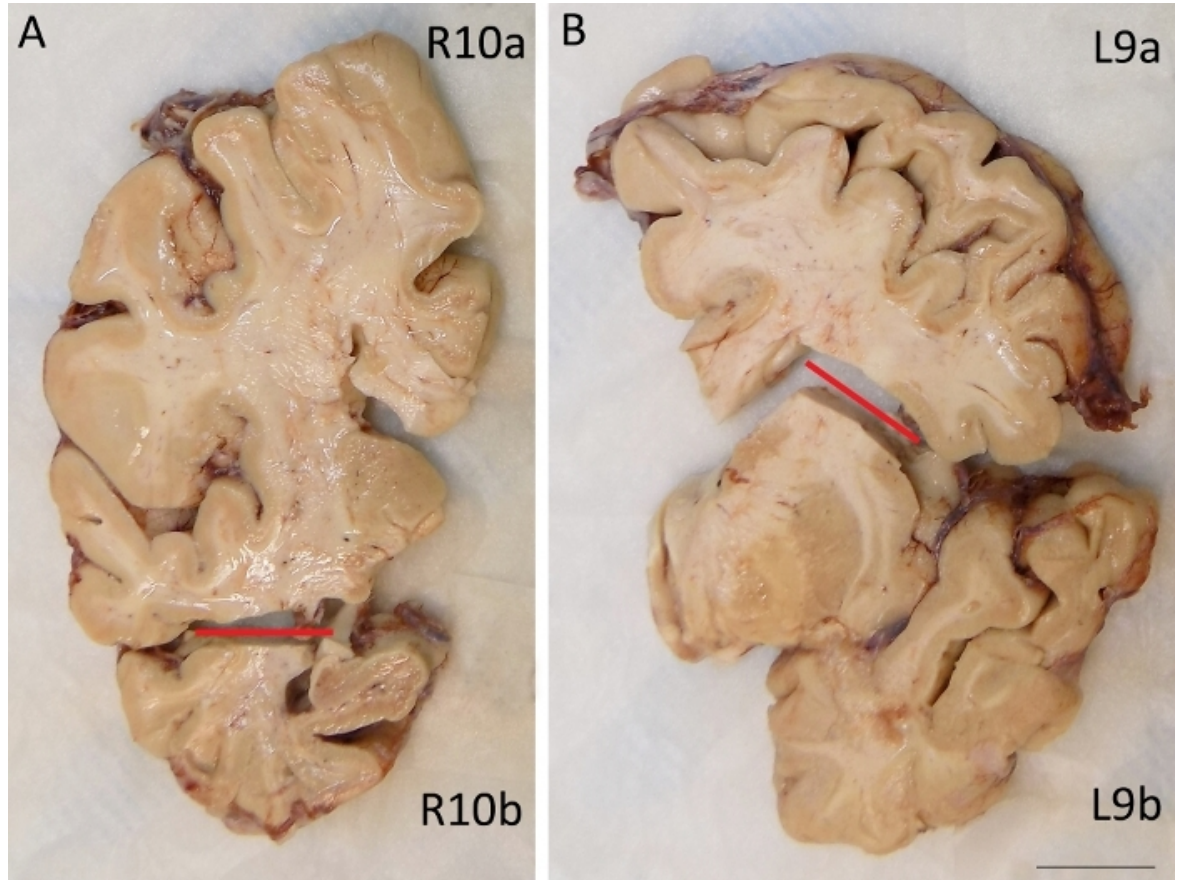

Figure 3: Coronal slices of fixed right $(A)$ and left $(B)$ fronto-temporal lobe are shown. Hippocampus and temporal lobe (A, R10b) are split from the frontal lobe (A, R10a) on the right slice. On the opposite slice, the amygdala and basal ganglia (B, L9b) are divided from the frontal lobe (B, L9a). Scale bar: $1.7 \mathrm{~cm}$. Please click here to view a larger version of this figure.

Relative Spectral Power Distribution Subject 16-1 NOLD

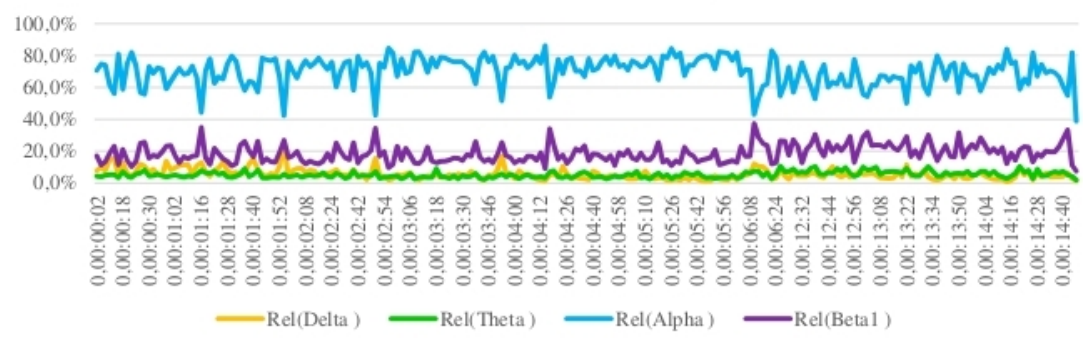

Relative Spectral Power Distribution Subject 189-15 Major-NCD

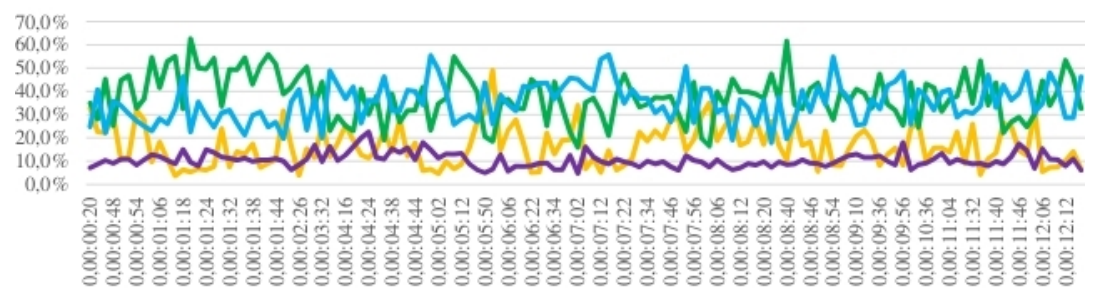

Figure 4: Relative spectral power distribution in NOLD and major-NCD subjects. Please click here to view a larger version of this figure. 

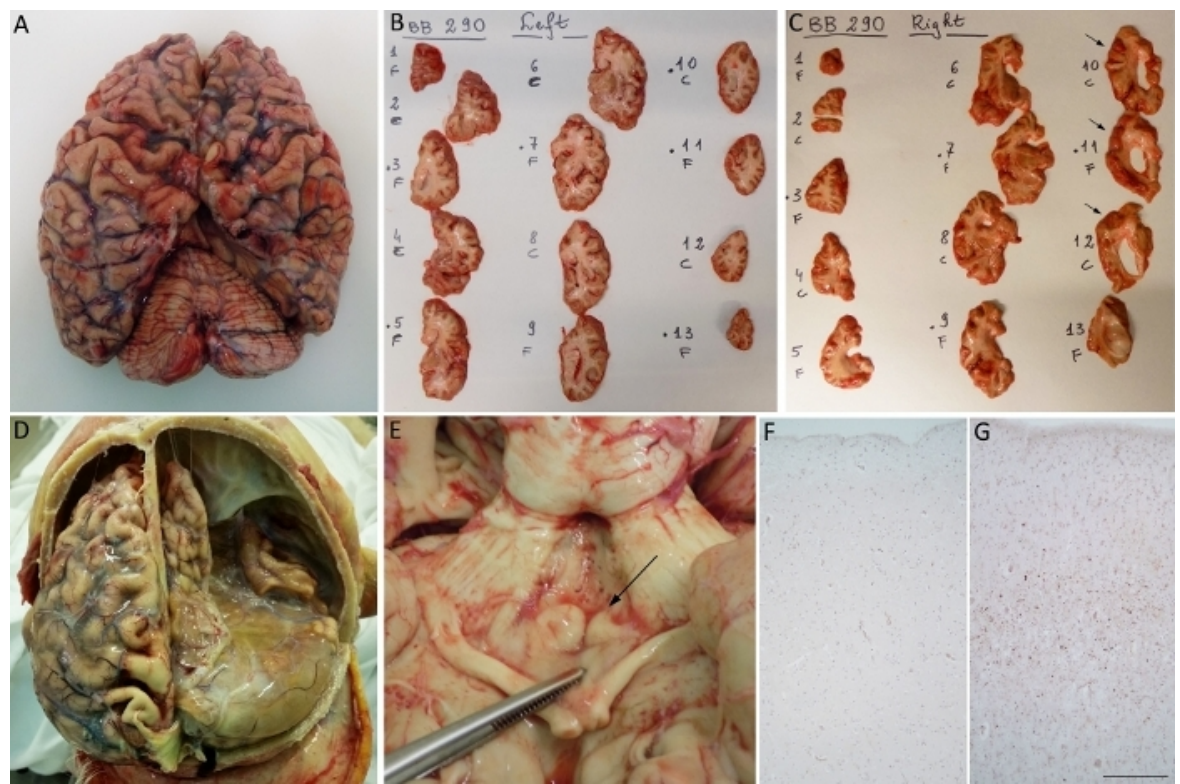

Figure 5: Representative images of asymmetric pathologies. (A) First case: brain with right atrophy. Coronal slices (B) and (C) show rightsided ventricular dilation particularly evident in sections 10-12 (C, arrows). In sections (B) and (C), "F" stands for fixed and "C" for frozen (in italian: congelato). (D) Second case: severe infarct of the right hemisphere. (E) Third case: atrophy of right mammillary body (arrow). (F, G) Fourth case: microscopic images show a more intense TDP-43 immunoreactivity in the frontal right cortex $(G)$ compared to the left one $(F)$ in a case of frontotemporal dementia. Scale bars: $183 \mu \mathrm{m}(\mathrm{F}, \mathrm{G})$. Please click here to view a larger version of this figure. 

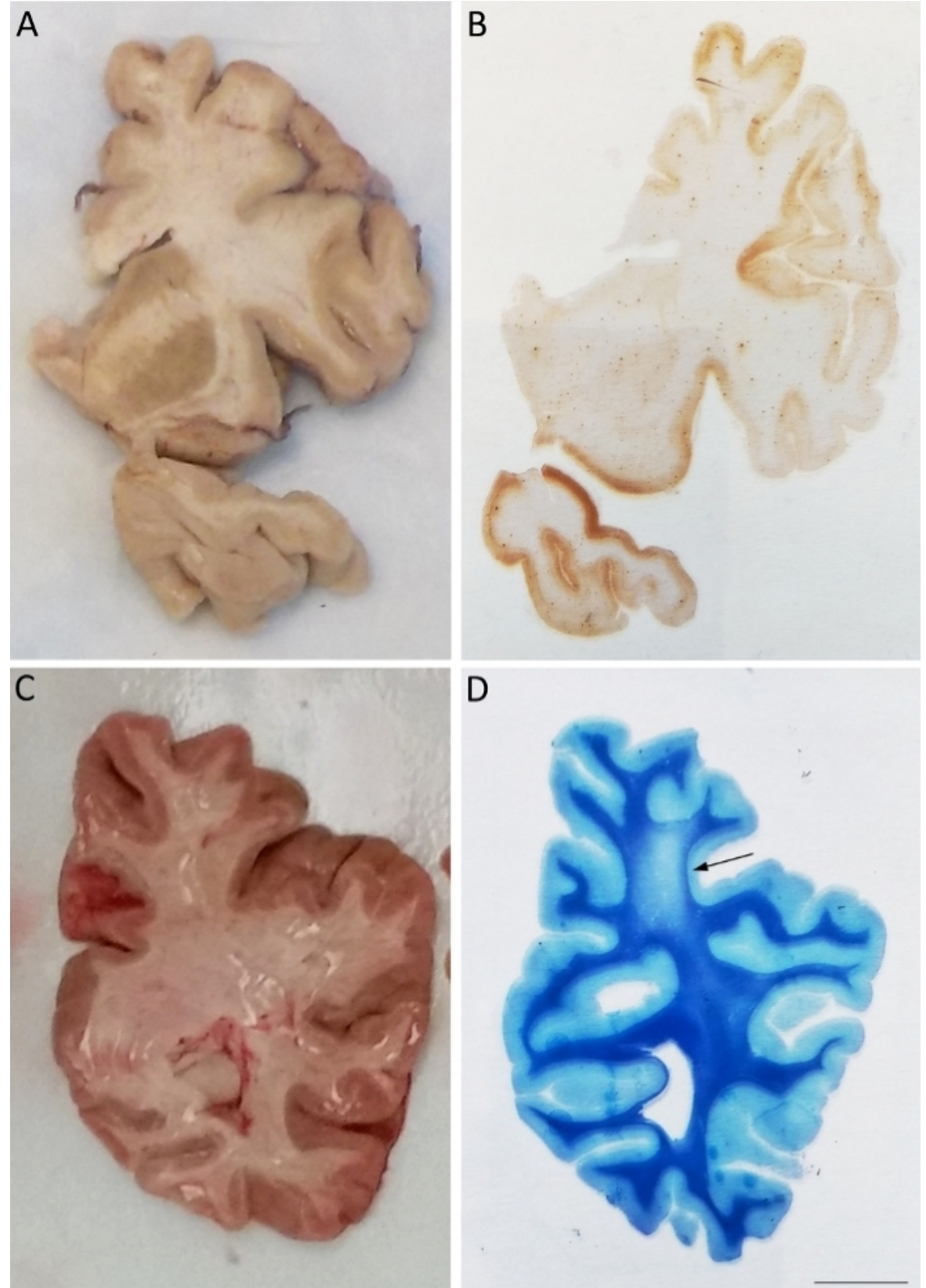

Figure 6: Macrosections. (A, C) Coronal slices of fixed fronto-temporal lobe (A) and fresh parietal lobe (C). (B) Histological fronto-temporal section immunolabeled with AT8 antibody. Immunoreactivity is clearly distributed all over the cortex, but more intense in the temporal lobe. (D) Histological parietal section stained with LFB. The arrow indicates an area of demyelination of the white matter. Scale bar: $1.55 \mathrm{~cm}(\mathrm{~B}, \mathrm{D})$. Please click here to view a larger version of this figure. 


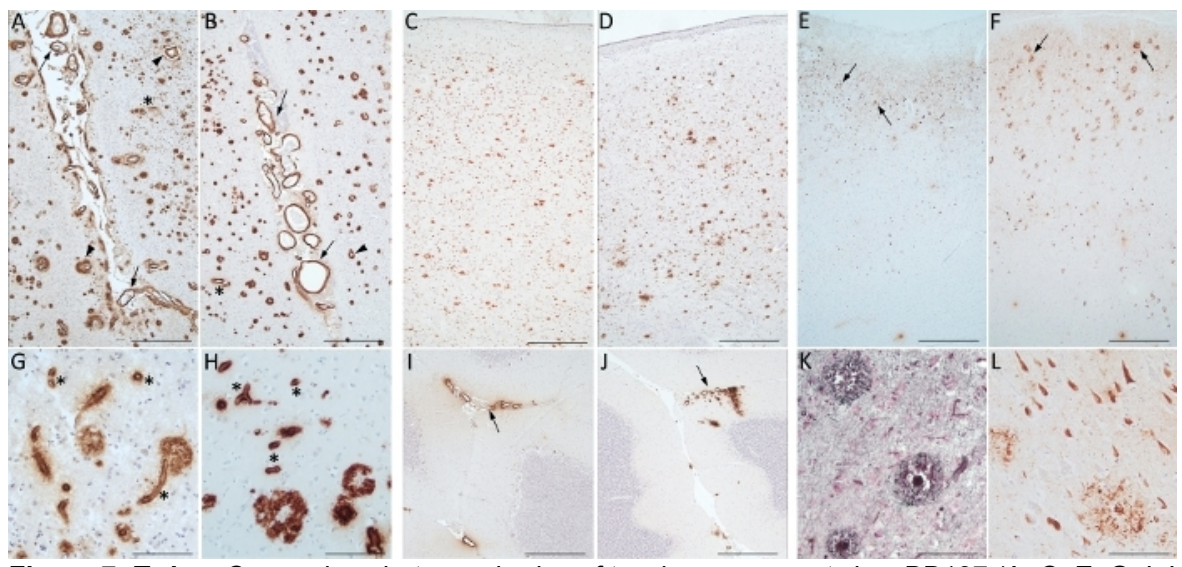

Figure 7: Twins. Comparison between brains of two homozygous twins: BB137 (A, C, E, G, I, K) and BB138 (B, D, F, H, J, L). The neuropathological pictures are very similar. (A) and (B) show diffuse 4G8 positivity in the occipital lobe with amyloid plaque, leptomeningeal (arrows) and parenchymal (arrowheads) vessels. Capillary amyloid angiopathy (asterisks) is well recognized at higher magnifications (G) and $(\mathbf{H}) .4 G 8$ is diffusely distributed throughout the basal ganglia (C, D) and around leptomeningeal vessels of cerebellum (I, J: arrows). AT8 immunoreactivity identify tangles, threads and plaques (arrows) in the parietal cortex (E, F). Gallyas staining (K) reveals neuritic plaques as AT8 antibody (L) does. Scale bars: $470 \mu \mathrm{m}(\mathrm{A}-\mathrm{F} ; \mathrm{I}-\mathrm{J}) ; 90 \mu \mathrm{m}(\mathrm{G}-\mathrm{H} ; \mathrm{K}-\mathrm{L})$. Please click here to view a larger version of this figure.
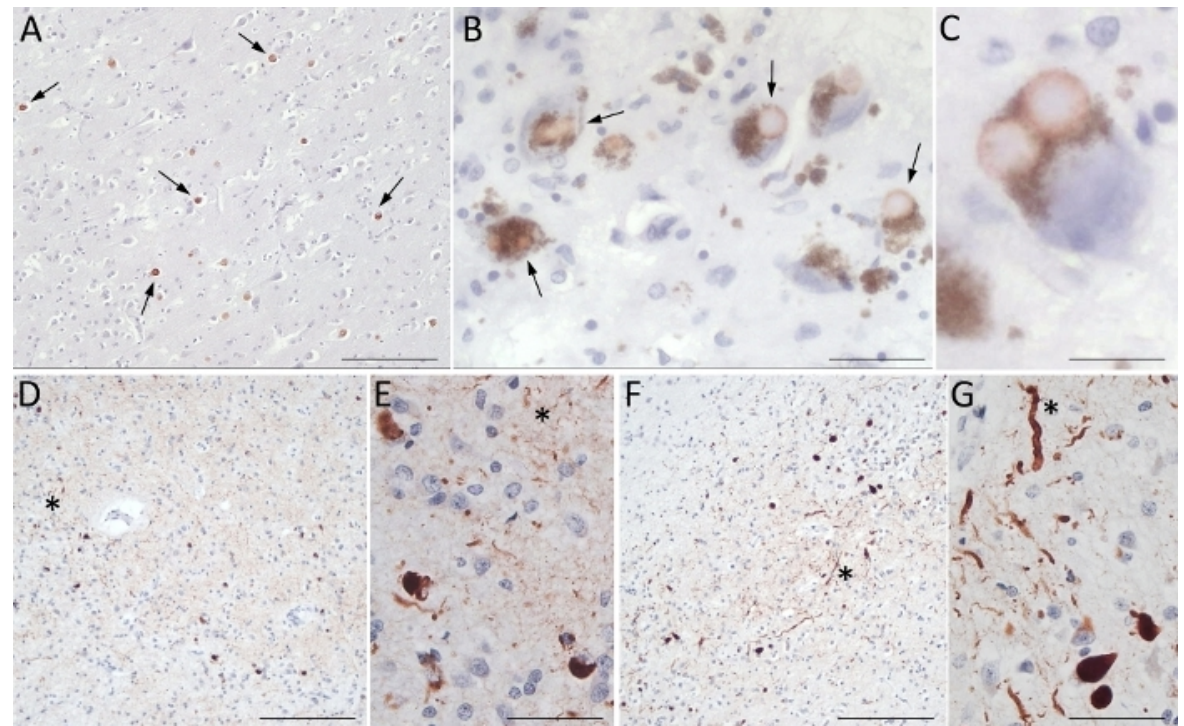

Figure 8: Clinical versus neuropathological diagnosis. In this figure, two different cases are reported in which the neuropathological diagnosis differs from the clinical diagnosis. Immunoreactivity for a-syn is an unexpected result. (A-C) First case: Gyrus cingoli (A) shows homogeneous distribution of Lewy bodies in $\mathrm{SN}$ (B-arrows). In a neuron of $\mathrm{SN}$, a double Lewy body surrounded by neuromelanin is shown (C). (D-G) Second case: A diffuse positivity for $\alpha$-syn is detectable in the amygdala (D, E) and Meynert nucleus ( $F, G)$. Cellular bodies and Lewy neurites (asterisk) are well marked. Scale bars: $154 \mu \mathrm{m}(\mathrm{A}, \mathrm{D}, \mathrm{F}) ; 37 \mu \mathrm{m}(\mathrm{B}, \mathrm{E}, \mathrm{G}) ; 20 \mu \mathrm{m}(\mathrm{C})$. Please click here to view a larger version of this figure. 


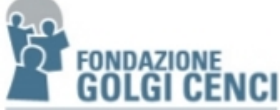

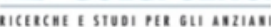

To the Director of

Golgi Cenci Foundation

Request for biological material

Applicant Information

First name, last name, professional qualification/position:
Fondazione Golgi Cenci

Corso San Martino 10

20081 Abbiategrasso

Fiscal code 90023310155 national research registry

(MIUR): 60125ADW T. +39.02 .94852265$ www.golgicenci.it

Affiliation:

Research Information

Title and brief description of the research project (attach the project protocol):

How the data/materials will be used within the research project:

Modality of publication/dissemination of results:

Detailed description and quantity of requested material

Responsible of the project:

First and Last Name:

e-mail:

Phone:

Address:

The Director of the Foundation must be informed about scientific publications resulting from the use of the requested data/material and the scientific contribution of the Brain Bank team must be taken into consideration for a possible co-authorship or acknowledgment. This application form is in line with the BNE code of conduct (Klioueva, 2018).

Signature

Date

Figure 9: Transfer agreement template. Please click here to view a larger version of this figure. 


\begin{tabular}{|c|c|c|c|}
\hline & & n & $\%$ \\
\hline \multirow[t]{2}{*}{ Gender } & Males & 607 & 45.9 \\
\hline & Females & 714 & 54.1 \\
\hline \multirow[t]{5}{*}{ Birth cohort } & 1935 & 236 & 17.8 \\
\hline & 1936 & 219 & 16.6 \\
\hline & 1937 & 264 & 20.0 \\
\hline & 1938 & 305 & 23.1 \\
\hline & 1939 & 297 & 22.5 \\
\hline \multirow[t]{5}{*}{ Marital status } & Married & 872 & 66.1 \\
\hline & Cohabiting & 13 & 1.0 \\
\hline & Separated/Divorced & 29 & 2.2 \\
\hline & Widowed & 325 & 24.6 \\
\hline & Single & 80 & 6.1 \\
\hline \multirow[t]{3}{*}{ Primary life occupation } & Blue collar workers & 666 & 50.6 \\
\hline & White collar workers & 459 & 34.9 \\
\hline & Housewife & 191 & 14.5 \\
\hline \multirow[t]{2}{*}{ Years of education } & $\leq 5$ years & 754 & 57.2 \\
\hline & $>5$ years & 565 & 42.8 \\
\hline
\end{tabular}

Table 1: Socio-demographic features of the InveCe.Ab study participants.

\begin{tabular}{|l|l|}
\hline INCLUSION CRITERIA & EXCLUSION CRITERIA \\
\hline All individuals aged 18 and above & People who blatantly refuse donation \\
\hline People living within the territory of Abbiategrasso & People who live outside the Lombardy region \\
\hline Volunteers who give consent on their own & $\begin{array}{l}\text { Discrepancies between the potential donor and NOKs wishes regarding } \\
\text { brain donation }\end{array}$ \\
\hline Subjects unable to decide with NOK's permission & Situations which greatly destroy the consistency of the brain \\
\hline Death by a natural cause & $\begin{array}{l}\text { Clinical course of <2 years unless the possibility of a prion disease has } \\
\text { been excluded }\end{array}$ \\
\hline & $\begin{array}{l}\text { Death caused by homicide or suicide, with the need for a coroner's } \\
\text { report }\end{array}$ \\
\hline & Post mortem interval >30 hours \\
\hline
\end{tabular}

Table 2: Criteria for brain donation. 


\begin{tabular}{|c|c|c|c|c|c|c|c|c|c|c|c|c|}
\hline $\mathbf{N}^{\circ}$ & $\operatorname{sex}$ & $\begin{array}{l}\text { CODE } \\
\text { BB }\end{array}$ & $\begin{array}{l}\text { CODE } \\
\text { InveCe }\end{array}$ & age & \begin{tabular}{|l} 
edu \\
(yrs)
\end{tabular} & clinical diagnosis & CDR & AFS & $\begin{array}{l}\text { PM } \\
\text { (hrs) }\end{array}$ & $\begin{array}{l}\mathrm{pH} \\
\text { tissue }\end{array}$ & $\begin{array}{l}\mathrm{pH} \\
\text { liquor }\end{array}$ & $\begin{array}{l}\text { neuropathological } \\
\text { diagnosis }\end{array}$ \\
\hline 1 & $F$ & BB 37 & & 87 & 5 & $\begin{array}{l}\text { Major-NCD due to AD } \\
\text { (BPSD) }\end{array}$ & 5 & 2 & 29 & nd & nd & $\begin{array}{l}\text { High AD pathology, } \\
\text { moderate SVD, } \\
\text { TDP43+, ctx LTS, HS }\end{array}$ \\
\hline 2 & $F$ & $\begin{array}{l}\text { BB } \\
105\end{array}$ & & 94 & 5 & Major-NCD due to AD & 5 & 1 & 5 & 5.72 & 6.78 & $\begin{array}{l}\text { High AD pathology, mild } \\
\text { SVD, HS }\end{array}$ \\
\hline 3 & $F$ & $\begin{array}{l}\text { BB } \\
137\end{array}$ & & 83 & 3 & $\begin{array}{l}\text { Major-NCD due to } \\
\text { multiple etiologies (AD- } \\
\text { VaD) }\end{array}$ & 5 & 1 & 16 & nd & nd & $\begin{array}{l}\text { High AD pathology, } \\
\text { moderate SVD, occipital } \\
\text { infarct, CAA-capCAA }\end{array}$ \\
\hline 4 & $M$ & $\begin{array}{l}\text { BB } \\
181\end{array}$ & & 71 & 13 & $\begin{array}{l}\text { Major-NCD due to AD } \\
\text { (BPSD) }\end{array}$ & 5 & 2 & 3 & nd & nd & $\begin{array}{l}\text { Severe LTS (Beach } \\
\text { IV), intermediate AD } \\
\text { pathology, mildSVD, } \\
\text { mCAA }\end{array}$ \\
\hline 5 & $M$ & $\begin{array}{l}\text { BB } \\
115\end{array}$ & 1636 & 78 & 18 & Major-NCD due to AD & 5 & 1 & 6 & nd & nd & $\begin{array}{l}\text { Intermediate AD, } \\
\text { moderate SVD, } \\
\text { Inflammation, ILBD } \\
\text { (Beach Ila), HS }\end{array}$ \\
\hline 6 & $F$ & BB 23 & 165 & 79 & 3 & $\begin{array}{l}\text { Major-NCD due to } \\
\text { vascular disease }\end{array}$ & 5 & 1 & 14 & nd & nd & $\begin{array}{l}\text { Severe and widespread } \\
\text { CAA, intermediate AD } \\
\text { pathology }\end{array}$ \\
\hline 7 & $M$ & $\begin{array}{l}\text { BB } \\
102\end{array}$ & 1412 & 79 & 8 & $\begin{array}{l}\text { Major-NCD due to } \\
\text { vascular disease }\end{array}$ & 3 & 1 & 8 & nd & nd & $\begin{array}{l}\text { Vascular Dementia, } \\
\text { ILBD }\end{array}$ \\
\hline 8 & $M$ & $\begin{array}{l}\text { BB } \\
224\end{array}$ & I 16 & 80 & 3 & $\begin{array}{l}\text { Major-NCD due to } \\
\text { multiple etiologies }\end{array}$ & 5 & 1 & 11 & nd & 5.99 & $\begin{array}{l}\text { Moderate SVD, Low AD } \\
\text { pathology, ILBD (Beach } \\
\text { Ila), HS }\end{array}$ \\
\hline 9 & $F$ & BB 47 & & 78 & 5 & $\begin{array}{l}\text { Major-NCD to multiple } \\
\text { etiologies (LBD-VaD } \\
\text { BPSD) }\end{array}$ & 5 & 0 & 8 & nd & nd & $\begin{array}{l}\text { High AD pathology, BG } \\
\text { TAU pathology, ARTAG, } \\
\text { mild SVD, HS }\end{array}$ \\
\hline 10 & $F$ & $\begin{array}{l}\text { BB } \\
153\end{array}$ & 1965 & 79 & 5 & $\begin{array}{l}\text { Mild-NCD (death due } \\
\text { to colon cancer with } \\
\text { widespread metastasis) }\end{array}$ & 0.5 & 1 & 8 & nd & 6.73 & $\begin{array}{l}\text { Low AD pathology, } \\
\text { moderate BG-SVD }\end{array}$ \\
\hline 11 & $M$ & $\begin{array}{l}\text { BB } \\
118\end{array}$ & I 1211 & 79 & 13 & $\begin{array}{l}\text { NOLD (death due to } \\
\text { liver cancer) }\end{array}$ & 0 & 2 & 3 & nd & 6.51 & Moderate SVD \\
\hline 12 & $M$ & $\begin{array}{l}\text { BB } \\
236\end{array}$ & 1521 & 80 & 3 & $\begin{array}{l}\text { Major-NCD due to AD } \\
\text { (BPSD) }\end{array}$ & 4 & 1 & 15 & nd & 6.15 & $\begin{array}{l}\text { High AD pathology, } \\
\text { Severe BG-SVD } \\
\text { (several microbleeds), } \\
\text { HS }\end{array}$ \\
\hline 13 & $F$ & $\begin{array}{l}\text { BB } \\
138\end{array}$ & & 85 & 3 & $\begin{array}{l}\text { Major-NCD due to } \\
\text { multiple etiologies (AD- } \\
\text { VaD BPSD) }\end{array}$ & 4 & 0 & 15 & nd & 6.75 & $\begin{array}{l}\text { High AD pathology, } \\
\text { moderate SVD, CAA, } \\
\text { limbic TDP43 }\end{array}$ \\
\hline 14 & $M$ & $\begin{array}{l}\text { BB } \\
109\end{array}$ & 1876 & 79 & 8 & $\begin{array}{l}\text { NOLD (death due to } \\
\text { brain tumor - GBL) }\end{array}$ & 0 & 1 & 16 & nd & 6.4 & $\begin{array}{l}\text { Low AD pathology, ILBD } \\
\text { (Beach Ila), mild SVD }\end{array}$ \\
\hline 15 & $F$ & $\begin{array}{l}\text { BB } \\
271\end{array}$ & & 84 & 8 & $\begin{array}{l}\text { Major-NCD due to AD } \\
\text { (BPSD) }\end{array}$ & 4 & 1 & 2 & nd & 6.7 & $\begin{array}{l}\text { Intermediate AD, limbic } \\
\text { LTS, moderate BG- } \\
\text { SVD, mCAA, TDP }\end{array}$ \\
\hline 16 & $F$ & BB 71 & I 1080 & 79 & 8 & $\begin{array}{l}\text { NOLD (death due to } \\
\text { heart failure) }\end{array}$ & 0 & 0 & 6 & nd & 6.26 & $\begin{array}{l}\text { Moderate BG-SVD, } \\
\text { ILBD, amy TDP, low AD }\end{array}$ \\
\hline 17 & $F$ & $\begin{array}{l}\text { BB } \\
189\end{array}$ & 1858 & 80 & 5 & $\begin{array}{l}\text { Major-NCD due to AD } \\
\text { (BPSD) }\end{array}$ & 3 & 0 & 20 & nd & 6.42 & $\begin{array}{l}\text { Intermediate AD, CAA- } \\
\text { capCAA, TDP43, } \\
\text { moderate BG-SVD, HS }\end{array}$ \\
\hline 18 & $F$ & $\begin{array}{l}\text { BB } \\
278\end{array}$ & 1924 & 80 & 5 & $\begin{array}{l}\text { Major-NCD due to LBD } \\
\text { (BPSD) }\end{array}$ & 3 & 1 & 5 & 6.02 & 7.05 & $\begin{array}{l}\text { Intermediate AD, limbic } \\
\text { LTS (Beach IV), limbic } \\
\text { TDP, HS }\end{array}$ \\
\hline 19 & $F$ & $\begin{array}{l}\text { BB } \\
247\end{array}$ & & 104 & 8 & $\begin{array}{l}\text { Major-NCD due to } \\
\text { multiple etiologies } \\
\text { (probably mixed } \\
\text { pathology) }\end{array}$ & 3 & 0 & 6 & 6.48 & 7.22 & $\begin{array}{l}\text { TAU pathology (PART- } \\
\text { ARTAG), HS }\end{array}$ \\
\hline
\end{tabular}




\begin{tabular}{|c|c|c|c|c|c|c|c|c|c|c|c|c|}
\hline 20 & $M$ & BB 85 & I 19 & 83 & 10 & $\begin{array}{l}\text { Major-NCD due to LBD } \\
\text { (BPSD) }\end{array}$ & 3 & 1 & 9 & 6.26 & 7.3 & $\begin{array}{l}\text { Severe limbic LTS, } \\
\text { intermediate AD, } \\
\text { Moderate SVD, severe } \\
\text { CAA-capCAA, HS }\end{array}$ \\
\hline 21 & $F$ & BB 14 & 1222 & 82 & 8 & $\begin{array}{l}\text { Major-NCD due to } \\
\text { multiple etiologies (AD- } \\
\text { VaD) }\end{array}$ & 2 & 1 & 11 & 5.59 & 6.4 & $\begin{array}{l}\text { Intermediate AD } \\
\text { pathology, Moderate } \\
\text { SVD }\end{array}$ \\
\hline 22 & $F$ & $\begin{array}{l}\text { BB } \\
282\end{array}$ & & 76 & 8 & $\begin{array}{l}\text { Major Frontotemporal } \\
\text { NCD (nfPPA BPSD) }\end{array}$ & 3 & 1 & 10 & 6.07 & 6.39 & $\begin{array}{l}\text { TDP (type A), ILBD, } \\
\text { moderate SVD, low AD, } \\
\text { HS }\end{array}$ \\
\hline 23 & $F$ & $\begin{array}{l}\text { BB } \\
154\end{array}$ & I 1079 & 80 & 5 & $\begin{array}{l}\text { NOLD (death due to } \\
\text { cancer with widespread } \\
\text { metastasis) }\end{array}$ & 0 & 1 & 5 & 6.49 & 6.9 & $\begin{array}{l}\text { moderate SVD, } \\
\text { CAA, low AD, limbic } \\
\text { encephalitis }\end{array}$ \\
\hline 24 & $F$ & $\begin{array}{l}\text { BB } \\
290\end{array}$ & & 65 & 13 & $\begin{array}{l}\text { major Frontotemporal } \\
\text { NCD (bvFTD BPSD) }\end{array}$ & 5 & 1 & 12 & 5.73 & 6.42 & TDP (type A) \\
\hline 25 & $F$ & $\begin{array}{l}\text { BB } \\
210\end{array}$ & & 89 & 8 & $\begin{array}{l}\text { Major-NCD due to AD } \\
\text { (BPSD) }\end{array}$ & 5 & 1 & 15 & 5.94 & 6.4 & in progress \\
\hline 26 & $M$ & $\begin{array}{l}\text { BB } \\
293\end{array}$ & & 75 & 18 & $\begin{array}{l}\text { Major Frontotemporal } \\
\text { NCD (bvFTD BPSD) }\end{array}$ & 5 & 1 & 8 & 6.14 & 6.83 & in progress \\
\hline 27 & $F$ & BB 99 & I 1370 & 79 & 9 & $\begin{array}{l}\text { NOLD (death due to } \\
\text { septic shock) }\end{array}$ & 0 & 2 & 14 & 6.3 & 7.12 & in progress \\
\hline & $M / F$ & & & mean & mean & & mean & mean & mean & mean & mean & \\
\hline & 0.5 & & & 81 & 7.7 & & 3.2 & 1.0 & 10.4 & 6.1 & 6.6 & \\
\hline
\end{tabular}

Table 3: Clinical/neuropathological diagnosis of ABB series. BB: Brain Bank; edu (yrs): educational years; CDR: Clinical Dementia Rating $(0=$ no dementia; 0.5 = mild cognitive impairment; 1 = mild dementia; 2 = moderate dementia; $3=$ severe dementia; $4=$ very severe dementia; 5 = terminal dementia); AFS: Agonal Factor Score; PM (hrs): Post Mortem hours; nd: not done; M/F: Males/Females; BPSD: Behavioral and Psychological Symptoms of Dementia; VaD: Vascular Dementia; NOLD: Normal elderly; GBL: Glioblastoma; nfPPA: non fluent Primary Progressive Aphasia; bvFTD: behavioral variant of Frontotemporal Dementia; SVD: Small Vessel Disease; LTS: Lewy Type Synucleinopathy; HS: Hippocampal Sclerosis; CAA: Cerebral Amyloid Angiopathy; capCAA: capillary CAA; mCAA: meningeal CAA; ILBD: Incidental Lewy Body

Disease; BG: Basal Ganglia; ARTAG: Age-Related TAU Astro-Gliopathy; PART: Primary Age-Related TAUopathy; amy: amigdala.

\begin{tabular}{|l|l|}
\hline Domain & Test name \\
\hline Depression & Center for Epidemiologic Studies Depression Scale (CES-D) [2] \\
\hline Global cognition & Mini-Mental State Examination (MMSE) [1] \\
\hline \multirow{2}{*}{ Verbal and visual Memory } & Free and Cued Selective Reminding Test [3] \\
\cline { 2 - 2 } & Corsi Test [4] \\
\cline { 2 - 3 } & Rey-Osterrieth Complex Figure (ROCF) Recall [5] \\
\hline Attention/ psychomotor speed & Trail making A [6] \\
\cline { 2 - 3 } & Attentional Matrices [4] \\
\hline \multirow{2}{*}{ Language-semantic memory } & Semantic Verbal Fluency (colours, animals, fruits, cities) [4] \\
\hline Executive functions & Trail making B [6] \\
\cline { 2 - 3 } & Raven's Coloured Matrices [7] \\
\hline Visuospatial abilities & Clock Drawing Test (CDT) [8] \\
\hline & Rey-Osterrieth Complex Figure (ROCF) Copy [5] \\
\hline
\end{tabular}

Table 4: Neuropsychological assessment for brain donors. 


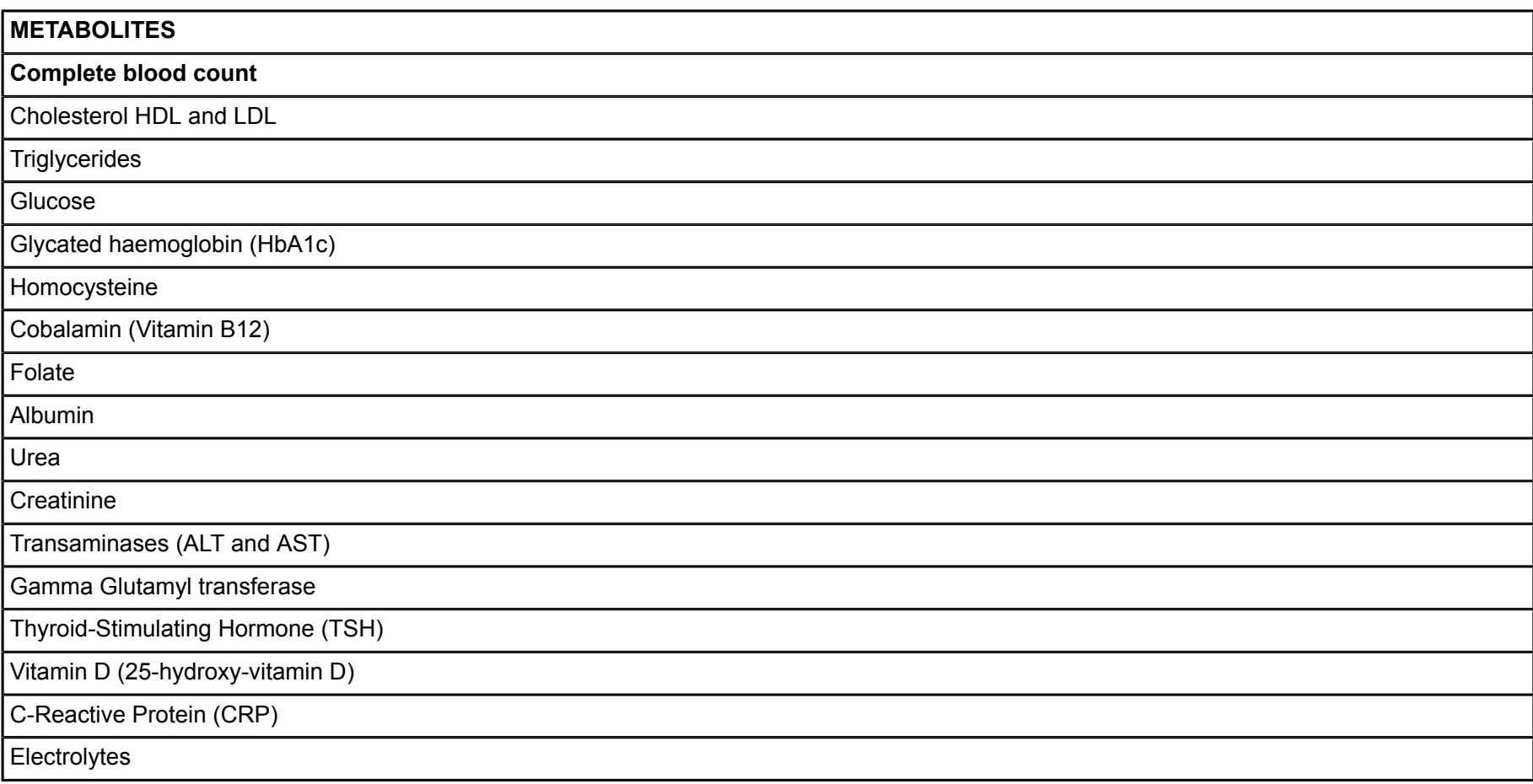

\section{Table 5: Metabolic panel for brain donors.}

\begin{tabular}{|l|l|}
\hline GENE NAME & dbSNP \\
\hline Apolipoprotein E (APOE) & rs429358 \\
\cline { 2 - 2 } & rs7412 \\
\hline Catalase (CAT) & rs1001179 \\
\hline Superoxide dismutase 2 (SOD2) & rs4880 \\
\hline Angiotensinogen (AGT) & rs699 \\
\hline Sirtuin 2 (SIRT2) & rs10410544 \\
\hline Translocase of outer mitochondrial membrane 40 (TOMM40) & rs2075650 \\
\hline Bridging integrator 1 (BIN1) & rs7561528 \\
\hline Catechol-O-methyltransferase (COMT) & rs4680 \\
\hline Methylenetetrahydrofolate reductase (MTHFR) & rs1801133 \\
\cline { 2 - 3 } & rs1801131 \\
\hline Brain derived neurotrophic factor (BDNF) & rs6265 \\
\hline solute carrier family 6, member 4 (SLC6A4 or 5HTT) & Hydroxytryptamine transporter gene-linked polymorphic region (5- \\
\cline { 2 - 2 } & HTTLPR) \\
\cline { 2 - 3 } & rs25531 \\
\hline
\end{tabular}

Table 6: SNPs analyzed for InveCe.Ab brain donors. 


\begin{tabular}{|c|c|c|c|}
\hline \multirow[t]{2}{*}{ PROCESS } & \multirow[t]{2}{*}{ SOLUTION } & \multicolumn{2}{|l|}{ DURATION } \\
\hline & & MACRO SAMPLES & MICRO SAMPLES \\
\hline FIXATION & 10\% BUFFERED FORMALIN & 8 days at $4^{\circ} \mathrm{C}$ & 8 days at $4{ }^{\circ} \mathrm{C}$ \\
\hline WASHING & PHOSPHATE BUFFER & $2-15$ days at room temperature & $2-15$ days at room temperature \\
\hline WASHING & $\mathrm{H}_{2} \mathrm{O}$ WASH & 2-3 h, tap water & 2-3 h, tap water \\
\hline DEHYDRATION & ETHYL ALCOHOL 70\% & $24 \mathrm{~h}$ & $8 \mathrm{~h}$ \\
\hline DEHYDRATION & ETHYL ALCOHOL 80\% & $24 \mathrm{~h}$ & $4 \mathrm{~h}$ \\
\hline DEHYDRATION & ETHYL ALCOHOL 90\% & $60 \mathrm{~h}$ (usually over the weekend) & $4 \mathrm{~h}$ \\
\hline DEHYDRATION & ETHYL ALCOHOL 95\% & $12 \mathrm{~h}$ & $4 \mathrm{~h}$ \\
\hline DEHYDRATION & ETHYL ALCOHOL 95\% & $12 \mathrm{~h}$ & $4 \mathrm{~h}$ \\
\hline DEHYDRATION & ETHYL ALCOHOL $100 \%$ & $6 \mathrm{~h}$ & $4 \mathrm{~h}$ \\
\hline DEHYDRATION & ETHYL ALCOHOL $100 \%$ & $6 \mathrm{~h}$ & $4 \mathrm{~h}$ \\
\hline CLEARING & XYLENE I & $12 \mathrm{~h}$ & $10 \mathrm{~h}$ \\
\hline CLEARING & XYLENE II & $12 \mathrm{~h}$ & $10 \mathrm{~h}$ \\
\hline INFILTRATION & PARAFFIN I & $12 \mathrm{~h}$ & $11 \mathrm{~h}$ \\
\hline INFILTRATION & PARAFFIN II & $12 \mathrm{~h}$ & $11 \mathrm{~h}$ \\
\hline EMBEDDING & PARAFFIN WAX & & \\
\hline
\end{tabular}

Table 7: ABB tissue processing protocol. 


\begin{tabular}{|c|c|c|c|c|c|c|c|c|c|c|}
\hline REGION & $H \& E$ & $\begin{array}{l}\text { CRESIL } \\
\text { VIOLET }\end{array}$ & LFB & GALLYAS & $4 \mathrm{G} 8$ & AT8 & $\alpha-S Y N$ & TDP-43 & NeuN & GFAP \\
\hline \multicolumn{11}{|l|}{ BRAINSTEM } \\
\hline $\begin{array}{l}\text { Medulla- } \\
\text { Dorsal } \\
\text { Motor } \\
\text { Nucleus of } \\
\text { Vagus }\end{array}$ & $x$ & & & & & $x$ & $x$ & & & \\
\hline $\begin{array}{l}\text { Pons - } \\
\text { Locus } \\
\text { Coeruleus }\end{array}$ & $x$ & & & & & $x$ & $x$ & & & \\
\hline \begin{tabular}{l|l} 
Midbrain - & xubstantia \\
Nigra & \\
Nigra
\end{tabular} & $x$ & & & & $x$ & $x$ & $x$ & & & \\
\hline \multicolumn{11}{|c|}{ CEREBELLUM } \\
\hline $\begin{array}{l}\text { Cerebellar } \\
\text { cortex and } \\
\text { Dentate } \\
\text { nucleus }\end{array}$ & $x$ & $x$ & $x$ & & $x$ & & & & & \\
\hline \multicolumn{11}{|l|}{ CEREBRUM } \\
\hline $\begin{array}{l}\text { Middle } \\
\text { frontal } \\
\text { gyrus }\end{array}$ & $x$ & $x$ & $x$ & & $x$ & $x$ & $x$ & $x$ & & \\
\hline $\begin{array}{l}\text { Basal } \\
\text { Ganglia + } \\
\text { nucleus of } \\
\text { Meynert }\end{array}$ & $x$ & $x$ & $x$ & & $x$ & & & & $x$ & $x$ \\
\hline $\begin{array}{l}\text { Cingulate, } \\
\text { anterior }\end{array}$ & $x$ & $x$ & $x$ & & $x$ & & $x$ & & $x$ & $x$ \\
\hline Amygdala & $x$ & & & & & $x$ & $x$ & $\mathrm{x}$ & $x$ & $\mathrm{x}$ \\
\hline \begin{tabular}{l|l} 
Thalamus \\
and \\
Subthalamic \\
nucleus
\end{tabular} & $x$ & & & & & $x$ & & & & \\
\hline \begin{tabular}{l|l} 
Superior \\
and middle \\
temporal \\
gyri
\end{tabular} & $x$ & $x$ & $x$ & $x$ & $x$ & $x$ & $x$ & & $x$ & $x$ \\
\hline $\begin{array}{l}\text { Hippocampuss } \\
\text { and } \\
\text { Entorhinal } \\
\text { cortex }\end{array}$ & sx & $x$ & $x$ & $x$ & $x$ & $x$ & $x$ & $x$ & $x$ & $x$ \\
\hline $\begin{array}{l}\text { Inferior } \\
\text { parietal } \\
\text { lobule }\end{array}$ & $\mathrm{x}$ & $\mathrm{x}$ & $x$ & $x$ & $x$ & $\mathrm{x}$ & $x$ & & & \\
\hline $\begin{array}{l}\text { Occipital } \\
\text { cortex }\end{array}$ & $x$ & & $x$ & & $x$ & $x$ & $x$ & & & \\
\hline $\begin{array}{l}\text { Olfactory } \\
\text { bulb }\end{array}$ & $x$ & & & & & & $x$ & & & \\
\hline
\end{tabular}

Table 8: Assessed regions, staining and immunohistochemistry.

\section{Discussion}

Critical steps in the protocol

Our aim is to obtain, characterize and store good quality tissues coming from subjects with detailed history derived from longitudinal observation. In order to reach this goal, it is required to deal with the following key aspects. As described above, the protocol begins with the recruitment of donors, which is the first crucial step. Then, it is necessary that the donors continue the follow-up program and maintain the adhesion to the project over time until the actual donation of the brain. At the time of death, it is necessary for the ABB staff to be promptly notified in order to convene the autopsy team within 24 hours, being important for adequate tissue quality. Fresh cutting of the cerebral hemispheres requires a 
steady hand and a specific training. To avoid cellular damage caused by slow freezing and obtain good quality slices for the cryostat and Omics, it is important that they are frozen quickly. Considering the speed of penetration of formalin solution ( $1 \mathrm{~mm} / \mathrm{hour})$, to preserve tissue antigenicity the soaking time of the single slice is kept at its minimum.

\section{Troubleshooting of the method}

In order to address the critical steps mentioned above we offer the following approach. Donor recruitment and adherence to follow-ups: There are several factors that hinder brain donation including fears of damaging the body's figure, purity and integrity, or of the possibility of feeling pain after death. Some even worry that the autopsy may be conducted whilst they are still alive ${ }^{70,71}$. Concerns about disruption of funeral arrangements and financial burden are also present ${ }^{72}$. Furthermore, a medical personnel's lack of knowledge about postmortem procedures and the inability to address the concerns of potential donors or their NOK may discourage registration. All these factors may produce a low level of awareness with a low number of enrolled participants and a high possibility of donor loss over time. Indeed, the donor recruitment program should be efficient in spreading awareness, instilling trust and convincing people to register and maintain high rates of follow-up participation. In our experience, this is done through careful selection of potential donors and thorough explanation of the BB's purposes. We offer educational activities and an empathic approach addressing the fears and needs of both healthy people and those affected with neurodegenerative diseases and their families. We find that potential donors are more likely to give their consent when approached in person. A face-to-face approach creates a relationship based on mutual trust and respect which is fundamental to achieving a high percentage of registration to brain donation and follow-up assessments. A highly trained staff with a strong sense of ethics first approaches the potential donor, discusses the possibility of donating the brain after death, explains the value of human brain tissue to neuroscientific research, and clarifies any doubt regarding postmortem procedures. Favorable word of mouth is equally important. After the brain harvesting, appropriate attention and care is given to recompose the cadaver. It is important to show respect and gratitude to the deceased person by treating the cadaver gently. A few months after, a meeting is scheduled to communicate the results of the neuropathological analysis whenever requested by family members.

The time of death and brain harvesting: When the person accepts, they become a donor and are given an identification card with a number to contact 24 hours/day, 7 days/week (the reception number of ASP Golgi-Redaelli Geriatric Hospital which is connected to us). Moreover, an adhesive tag is given to the relatives to be used in case of hospitalization. The funeral agencies of the area were previously informed to bring the body to the ABB facilities, where the autopsy team is summoned. The autopsy team is composed of a pathologist, a neurologist and/or a neurobiologist, and an anatomical room technician, which are on call from 6 am to $11 \mathrm{pm}$ every day; some trainee students are also frequently present to assist and take pictures.

The precision and consistency of the fresh cutting procedures are ensured by the involvement of the same two operators (a neurologist and a pathologist) who have developed the method and have several years of experience in neuropathology. When freezing the slices, they are put on a prefrozen aluminum tray and covered with an interlocking aluminum plate to keep them well flat. Immediately after, they are put in liquid nitrogen for 3 minutes, before storing them at $-80^{\circ} \mathrm{C}$. The slices to be fixed are individually wrapped in gauze, and soaked into a $10 \%$ phosphate buffered formalin solution, which is substituted after one day. Subsequently, they are kept in formalin no more than 5 additional days; however, considering that the formalin solution penetrates at $1 \mathrm{~mm} /$ day, we would like to shorten furtherly the soaking time.

\section{Limitations of the method}

The research method described here only covers a limited geographical area, and the individuals involved in the donation program possess characteristics that do not entirely represent the general population. Although more than acceptable, a postmortem interval up to 24 hours may produce alterations in some protein structures, enzymes and RNA of brain tissue. The determination of AFS and pH may not be entirely adequate for determining tissue quality ${ }^{73}$ and we are developing other ways of authenticating tissue quality based on RNA integrity.

Concerning the microtome cutting procedure, although very useful for reconstructing anatomical relationships, it should be noted that the use of macrosections is not simple and presents some technical difficulties. Our method is challenging and time-consuming. The costs are quite high and funding is not always easy. The funding comes primarily from public (ASP Golgi-Redaelli Geriatric Hospital) and private resources (GolgiCenci Foundation), private donations, non-profit organizations (e.g. "Federazione Alzheimer Italia") and grants participation.

\section{The significance of the ABB method with respect to existing/alternative methods}

At the beginning, our brain donation program targeted individuals participating in the InveCe.Ab longitudinal study. As a consequence, the brains donated are accompanied by detailed clinical, biological and social information collected over the years. The strength of ABB derives precisely from this distinctive origin. Indeed, studying a group of socially and genetically related people who share biological characteristics and environmental exposures enhances statistical analysis. Moreover, the study includes the following benefits: 1) Looking after and providing for the needs of the community (the act of "giving before asking"): people receive a free periodic check-up of which the general practitioner is informed, a telephone number of our secretary is provided for consultations, and severely disabled people are visited at home; 2) Engaging participants, people with public roles and general practitioners in educational activities through the organization of periodic seminars (related to brain healthiness, brain donation and general health), and the planning of thematic courses (e.g. the use of information technology devices for elderly); 3) Meeting people and adopting a face-to-face approach. All these elements constitute the strength of the ABB project. Furthermore, the project improves the clinical abilities of involved medical personnel, as they gain experience in both antemortem assessments and postmortem neuropathological evaluations. In this regard, we would like to mention the very peculiar experience of "The minority aging research study". This study involved a limited and selected number of African Americans residing in the Chicago area (784 out of 1,357 eligible subjects: response rate of $57 \%$ ). The participants were visited annually at home and were asked to join the brain donation program. This study is based on an unusual approach with some similarities to ours obtaining high percentages of positive response to the brain donation program (352 donors out of 784 participants were enrolled: $44 \%$ ), albeit the autopsy rate was not quite satisfactory $(53 \%)^{74}$. Just like in "The minority aging research study", we offer educational activities and an empathic approach, achieving excellent results. In particular, our response rate is $93 \%$, the enrollment percentage is $28.7 \%$, and the autopsy rate at the moment is $67 \%$. These rates show that projects directly engaging people have a more substantial percentage of donations. Other brain donation programs, with less attention into building a relationship with potential donors, generally have a low enrollment percentage, being around $10-15 \%$ or less ${ }^{73,75}$.

There are few previous cohort studies ending with a neuropathological analysis. Moreover, the majority of brain banks and repositories are disease-centered and there is a scarcity of "control" brains from healthy donors compared to the number of "diseased" brains. Only a limited number of BBs are based on population studies involving both diseased and normal subjects in order to study aging trajectories ${ }^{73,74,76,77,78,79,80}$. 
Some studies such as "The minority aging research study" in the USA ${ }^{74}$ and "The Vantaa $85+$ study" in Finland ${ }^{76}$ are similar to ours but they tend to run out with the termination of the cohort. Instead, ABB's donation program is envisioned to continue for a long time in the future, enlisting potential donors and scheduling follow-ups even after the end of the InveCe.Ab longitudinal study. This approach makes our recruitment method similar to that of the "Sun Health Research Institute (SHRI) brain donation program" which is aimed at a retirement community ${ }^{73}$. The SHRI protocol for brain donation is very efficient with the shortest mean postmortem interval in the world (3.92 hours). Similar to the biggest BBs in the world, the SHRI protocol simply cut the cerebrum, cerebellum and brainstem in the midline (sagittal plane), then one half is dissected fresh and frozen for biochemical studies, while the other is fixed in formalin for the histopathological assessment. However, one of the strengths of the SHRI dissection protocol is the fixation of individual slices instead of the whole hemisphere. Indeed, fixating a hemisphere as a whole is not optimal, because of the different fixation gradients between the surface and the core. Moreover, the cortical proteins may be affected by prolonged exposure to the formalin solution. Thus, the reason why we decided to fix individual slices.

The decision as to which side is fixed or frozen, depends on the singular bank (always the same, randomly assigned or assigned depending on whether the day of dissection is odd or even $)^{31,32,33,34,35}$. So, biochemical and histopathological analysis are conducted separately on each hemisphere. As many neurological diseases are asymmetrical, our BB offers a unique protocol for slicing fresh specimens: alternate sections from the brainstem and from each hemisphere of cerebellum and cerebrum are retained as fixed or frozen material; a fixed slice on one hemisphere corresponds to a frozen one on the other hemisphere. Our method gives the opportunity to obtain a complete histological characterization of all frozen material and to compare the results from all areas of both sides. As said in the introduction, the method described allows us to obtain as much information as possible from the brain tissue. Furthermore, ABB's method yields a basic but complete neuropathological characterization, including almost all known brain proteinopathies and vascular pathology. Due to the controversial role of vascular injuries in determining cognitive impairment, we decided to use a double scoring for the vascular burden ${ }^{30,53}$.

As explained in the protocol, we use a multidisciplinary approach. Although this is a time-consuming and labored method, we believe it provides several advantages for research. For instance, in a previous work, we demonstrated that high tHcy per se, or MTHFR C677T TT associated with the APOE- $\varepsilon 4$ allele, may be related to executive dysfunctions rather than memory loss ${ }^{81}$. Therefore, it may be interesting to evaluate subjects with this particular genetic profile at a neuropathological level. This is an example of how such in-depth follow-up is useful in creating new research hypotheses verifiable through investigation of biological material collected in our bank. Another demonstration of the advantage of our approach is the inclusion of QEEG among the assessments we routinely perform, for its originality and the relative ease of use. Indeed, EEG detects the synaptic activity of the cerebral cortex by recording the electrical potentials of dendrites belonging to cortical pyramidal neurons ${ }^{82}$. QEEG can be considered a biomarker for estimation of cortical synaptic activity which is related to cognition ${ }^{83}$. Particularly, a decrease in alpha rhythms in the posterior part of the brain with a general increase of lower frequencies (theta and delta rhythms) has been related to cortical connection breakdown. It should be considered that most of the diagnostic correlation studies have been based on a clinical diagnosis that is only a probable and not a definite diagnosis ${ }^{84,85,86,87}$. Only very few targeted studies have compared QEEG data with the neuropathological picture to investigate the correlation between QEEG and LTS variants ${ }^{88,89}$, and the distinction between FTLD and $A D^{83}$. By ending our study with a definition of the neuropathological diagnosis, it is then possible to correctly interpret the observations made on the cerebral electrical activity. Moreover, performing serial QEEG in each subject we can track intra-individual EEG waves trajectory and their correlations with the neuropathological picture. Following individual modifications of cortical electrical activity over time can lead to a better understanding of its meaning as a biomarker for incipient dementia.

\section{Future applications and direction of the method}

Implementing the tissue distribution is one of our main prospective goals. In order to do this, we just established a scientific commission including the director of the GC Foundation (geriatrician), an academic of Neurology from the University of Pavia, a neurologist and a pathologist both from the GC Foundation \& the Geriatric Hospital ASP Golgi-Redaelli. When distributing brain tissue, histological slides, and other biological samples, it is important to remember that donors agreed to the donation for the sake of research. The distribution of material should therefore take place prudently, just as described in the BNE Code of Conduct ${ }^{40}$. Any party submitting a request for material should indicate the type and quantity of the sample needed, provide a description of the research project and how the samples will be used, and whenever possible, supply evidence of previous publications (for the transfer agreement, see Figure 9). The brain bank does not work for financial gain. So, the fees paid by researchers should only cover the expenses of the tissue procurement, processing, storage and distribution.

Plans to start analyzing cases with exome sequencing and Omics techniques, such as proteomics and transcriptomics, are in motion. Our alternate sampling methodology will allow omics studies to be performed on histologically well-defined tissues from both hemispheres.

Through this approach, it will be possible to compare the pattern of gene activation in different brain areas of the same hemisphere and in the corresponding areas of the other hemisphere, and be able to correlate gene activation with histopathology. In this field, deep learning applications would be of great interest including computerized analysis of histological slides and cutting-edge correlation of clinical, histological and omics data. Other correlations between many different variables can be identified as well as other possible technological applications. The availability of frozen material from both hemispheres will allow an accurate topography of gene activation and protein distribution. This will be of particular interest even in healthy subjects, considering that both brain functions and some diseases are asymmetrical.

Moreover, we can obtain cell cultures from well-characterized brain donors. Indeed, cell cultures from the leptomeninges of harvested brains supply living cells that can be used for further investigation of disease or aging mechanisms, by means of the induced pluripotent stem cells (iPSCs) technology which involves reprogramming leptomeningeal fibroblasts and differentiating them into neural cells in advanced models ${ }^{90}$.

As brains age, different profiles of change can occur at the molecular, cellular and tissue level. Every brain is unique. Each has its own way of responding to internal and external stressors; some resists while others succumb and display distinct pathologies. Discrepancies between the clinical presentation and the neuropathological picture often exist because the topography of the lesions, rather than their molecular nature, determines the clinical presentation. The correct and definite diagnosis could only be achieved by combining the clinical syndrome with the neuropathological findings which often add important etiological clues necessary to unravel the pathogenesis of the diseases. In Europe, there is an effort to create a standard approach to neuropathological diagnosis. Our diagnostic protocol almost completely follows the recently published guidelines on neuropathological diagnosis for brain banking ${ }^{91}$. This will allow us to collect and share well-documented brain tissue, with the prospective goal of establishing the first Italian Brain Bank. Indeed, in Italy there are brain repositories but not brain banks based on cohort studies. Our aim is to develop a method for brain tissue harvesting that can be implemented broadly across Italy, to establish a network that uses 
a common protocol and shares comparable material. In order to do so, the involvement of other research centers and the creation of a specific website are among the main objectives for the future.

Innovative technologies are constantly being used for the analysis of the molecular nature of neurodegenerative diseases and for biomarker identification. In this context, there will be an increasing need for brains accompanied by information about cognitive and aging trajectories obtained through longitudinal studies, emphasizing the importance of a neuropathologically verified epidemiological approach ${ }^{92}$.

\section{Disclosures}

The authors have nothing to disclose.

\section{Acknowledgments}

We wish to dedicate this work to Dr.ssa Michela Mangieri. Before she died prematurely, she conceived and started the Abbiategrasso Brain Bank Project.

We are grateful to our brain donors, who are generously contributing to research donating the noblest organ of their body; without them this research would not be possible.

We are grateful to Valeria Marzagalli for her precious work in the ABB project.

The authors thank Prof. Johannes Attems, Dr. Paolo Fociani and Dr. Giorgio Giaccone for their precious guidance and wise counsel.

We would like to thank Dr.ssa Alice Cirrincione and Miss Giulia Bortone for their precious help throughout the project.

Many thanks to Mrs. Tere Cassani for her support and to "Federazione Alzheimer Italia" for its collaboration.

The authors are grateful to Dr. Matteo Moretti and Prof. Antonio Marco Maria Osculati, Department of Public Health, Experimental and Forensic

Medicine, University of Pavia.

\section{References}

1. The Global Dementia Observatory Reference Guide World Health Organization. at <http://apps.who.int/bookorders> (2018).

2. Kasper, B.S. et al. Neuropathology of epilepsy and psychosis: the contributions of J.A.N. Corsellis. Brain: a journal of neurology. 133 (Pt 12), 3795-805 (2010).

3. Overy, C., Tansey, E.M. The development of brain banks in the UK. c.1970-c.2010. at <www.histmodbiomed.org>. (2013).

4. Tourtellotte, W.W., Itabashi, H.H., Rosario, I., Berman, K. The National Neurological Research Bank. A collection of cryopreserved human neurological specimens for neuroscientists. Annals of the New York Academy of Sciences. 436, 513-6 (1984).

5. Tourtellotte, W.W., Rosario, I.P., Conrad, A., Syndulko, K. Human neuro-specimen banking 1961-1992. The National Neurological Research Specimen Bank (a donor program of pre- and post-mortem tissues and cerebrospinal fluid/blood; and a collection of cryopreserved human neurological specimens for neuroscientists). Journal of neural transmission. Supplementum. 39, 5-15 (1993).

6. Carlos, A.F., Poloni, T.E., Medici, V., Chikhladze, M., Guaita, A., Ceroni, M. From brain collections to modern brain banks: A historical perspective. Alzheimer's \& dementia (New York, N. Y.). 5, 52-60 (2019).

7. Szymański, P., Markowicz, M., Janik, A., Ciesielski, M., Mikiciuk-Olasik, E. Neuroimaging diagnosis in neurodegenerative diseases. Nuclear medicine review. Central \& Eastern Europe. 13 (1), 23-31 (2010).

8. Nowak, D., Hofmann, W.K., Koeffler, H.P. Genome-Wide Mapping Of Copy Number Variations using SNP Arrays. Transfusion Medicine and Hemotherapy. 36 (4), 246-251 (2009).

9. Bush, W.S., Moore, J.H. Chapter 11: Genome-wide association studies. PLoS computational biology. 8 (12), e1002822 (2012).

10. Dirks, R.A.M., Stunnenberg, H.G., Marks, H. Genome-wide epigenomic profiling for biomarker discovery. Clinical epigenetics. 8 (1), 122 (2016).

11. Felsenfeld, G. A Brief History of Epigenetics. Cold Spring Harbor Perspectives in Biology. 6 (1), a018200-a018200 (2014).

12. Cogswell, J.P. et al. Identification of miRNA Changes in Alzheimer's Disease Brain and CSF Yields Putative Biomarkers and Insights into Disease Pathways. Journal of Alzheimer's Disease. 14 (1), 27-41 (2008).

13. Lau, P. et al. Alteration of the microRNA network during the progression of Alzheimer's disease. EMBO molecular medicine. 5 (10), 1613-34 (2013).

14. Lowe, R., Shirley, N., Bleackley, M., Dolan, S., Shafee, T. Transcriptomics technologies. PLoS computational biology. 13 (5), e1005457 (2017).

15. Simpson, J.E. et al. Microarray analysis of the astrocyte transcriptome in the aging brain: relationship to Alzheimer's pathology and APOE genotype. Neurobiology of aging. 32 (10), 1795-807 (2011).

16. Shevchenko, G., Konzer, A., Musunuri, S., Bergquist, J. Neuroproteomics tools in clinical practice. Biochimica et biophysica acta. 1854 (7), 705-17 (2015)

17. Patterson, S.D. Proteomics: evolution of the technology. BioTechniques. 35 (3), 440-4 (2003).

18. Paraizo Leite, R.E., Tenenholz Grinberg, L. Closing the gap between brain banks and proteomics to advance the study of neurodegenerative diseases. Proteomics. Clinical applications. 9 (9-10), 832-7 (2015).

19. Giacomelli, C., Daniele, S., Martini, C. Potential biomarkers and novel pharmacological targets in protein aggregation-related neurodegenerative diseases. Biochemical Pharmacology. 131, 1-15 (2017).

20. Faghihi, M.A., Mottagui-Tabar, S., Wahlestedt, C. Genetics of neurological disorders. Expert review of molecular diagnostics. 4 (3), 317-32 (2004).

21. Toft, M. Advances in genetic diagnosis of neurological disorders. Acta Neurologica Scandinavica. 129, $20-25$ (2014). 
22. Kumar, D., Weatherall, D.J. Genomics and clinical medicine. 651 (2008).

23. Han, G., Sun, J., Wang, J., Bai, Z., Song, F., Lei, H. Genomics in Neurological Disorders. Genomics, Proteomics \& Bioinformatics. 12 (4), 156-163 (2014).

24. Gere, C. A Brief History of Brain Archiving. Journal of the History of the Neurosciences. 12 (4), 396-410 (2003).

25. Fratiglioni, L., Paillard-Borg, S., Winblad, B. An active and socially integrated lifestyle in late life might protect against dementia. Lancet neurology. 3 (6), 343-353 (2004).

26. Spartano, N.L. et al. Association of Accelerometer-Measured Light-Intensity Physical Activity With Brain Volume. JAMA Network Open. 2 (4), e192745 (2019).

27. Bell, J.E. et al. Management of a twenty-first century brain bank: experience in the BrainNet Europe consortium. Acta neuropathologica. 115 (5), 497-507 (2008).

28. Ravid, R., Park, Y. mok Brain banking in the twenty-first century: creative solutions and ongoing challenges. Journal of Biorepository Science for Applied Medicine. 2, 17 (2014).

29. Iacono, D., Geraci-Erck, M., Peng, H., Bouffard, J.P. Symmetric Bihemispheric Postmortem Brain Cutting to Study Healthy and Pathological Brain Conditions in Humans. Journal of visualized experiments: JoVE. (118) (2016).

30. Deramecourt, V. et al. Staging and natural history of cerebrovascular pathology in dementia. Neurology. 78 (14), 1043-50 (2012).

31. Netherlands Brain Bank Information for tissue applicants. at <https://www.brainbank.nl/media/uploads/file/Information for tissue applicants_2019.pdf>. (2019).

32. UK Brain Bank Network Process used by the banks for tissue processing and storage. The UK Brain Bank Network protocol. at <https:// mrc.ukri.org/documents/pdf/process-used-by-the-banks-for-tissue-processing-and-storage/>. (2019).

33. Vonsattel, J.P.G., Del Amaya, M.P., Keller, C.E. Twenty-first century brain banking. Processing brains for research: the Columbia University methods. Acta neuropathologica. 115 (5), 509-32 (2008).

34. Brain Bank, H. A Tour Of The Brain Bank. at <https://hbtrc.mclean.harvard.edu/pdf/about/HBTRC-Tour-2013.2.pdf>. (2013).

35. Sheedy, D. et al. An Australian Brain Bank: a critical investment with a high return! Cell and tissue banking. 9 (3), $205-16$ (2008).

36. Guaita, A. et al. Brain aging and dementia during the transition from late adulthood to old age: design and methodology of the "Invece.Ab" population-based study. BMC Geriatr. 13, 98 (2013).

37. Lobo, A. et al. Prevalence of dementia and major subtypes in Europe: A collaborative study of population-based cohorts. Neurologic Diseases in the Elderly Research Group. Neurology. 54, S4-9 (2000).

38. Hofman, A. et al. The prevalence of dementia in Europe: a collaborative study of 1980-1990 findings. Eurodem Prevalence Research Group. Int J Epidemiol. 20 (3), 736-48 (1991).

39. BrainNet Europe - Code of Conduct. at <https://www.brainnet-europe.org/indexe151.html? option=com_content\&view=article\&id=89\&Itemid=89> (2008).

40. Klioueva, N.M., Rademaker, M.C., Huitinga, I. Design of a European code of conduct for brain banking. Handbook of Clinical Neurology. 150 , Elsevier B.V. (2018).

41. Lee, K., Saetern, O.C., Nguyen, A., Rodriguez, L., Schüle, B. Derivation of Leptomeninges Explant Cultures from Postmortem Human Brain Donors. Journal of visualized experiments: JoVE. (119) (2017).

42. Esiri, M.M., Wilcock, G.K., Morris, J.H. Neuropathological assessment of the lesions of significance in vascular dementia. Journal of Neurology, Neurosurgery \& Psychiatry. 63 (6), 749-753 (1997).

43. Tomita, $\mathrm{H}$. et al. Effect of agonal and postmortem factors on gene expression profile: quality control in microarray analyses of postmortem human brain. Biological psychiatry. 55 (4), 346-52 (2004).

44. Stan, A.D. et al. Human postmortem tissue: what quality markers matter? Brain research. 1123 (1), 1-11 (2006).

45. Montine, T.J. et al. National Institute on Aging-Alzheimer's Association guidelines for the neuropathologic assessment of Alzheimer's disease: a practical approach. Acta Neuropathologica. 123 (1), 1-11 (2012).

46. Boxer, A.L., Yu, J.T., Golbe, L.I., Litvan, I., Lang, A.E., Höglinger, G.U. Advances in progressive supranuclear palsy: new diagnostic criteria, biomarkers, and therapeutic approaches. The Lancet Neurology. 16 (7), 552-563 (2017).

47. Dickson, D.W., Ahmed, Z., Algom, A.A., Tsuboi, Y., Josephs, K.A. Neuropathology of variants of progressive supranuclear palsy. Current opinion in neurology. 23 (4), 394-400 (2010).

48. Dickson, D. Neurodegeneration: the molecular pathology of dementia and movement disorders. Wiley-Blackwell. (2011).

49. Crary, J.F. et al. Primary age-related tauopathy (PART): a common pathology associated with human aging. Acta neuropathologica. 128 (6), 755-66 (2014).

50. Kovacs, G.G. et al. Aging-related tau astrogliopathy (ARTAG): harmonized evaluation strategy. Acta neuropathologica. 131 (1), 87-102 (2016).

51. Alafuzoff, I. et al. Neuropathological assessments of the pathology in frontotemporal lobar degeneration with TDP43-positive inclusions: an inter-laboratory study by the BrainNet Europe consortium. Journal of neural transmission (Vienna, Austria: 1996). 122 (7), 957-72 (2015).

52. Love, S. et al. Development, appraisal, validation and implementation of a consensus protocol for the assessment of cerebral amyloid angiopathy in post-mortem brain tissue. American journal of neurodegenerative disease. 3 (1), 19-32 (2014).

53. Skrobot, O.A. et al. Vascular cognitive impairment neuropathology guidelines (VCING): the contribution of cerebrovascular pathology to cognitive impairment. Brain. 139 (11), 2957-2969 (2016).

54. Thal, D.R., Rüb, U., Orantes, M., Braak, H. Phases of A beta-deposition in the human brain and its relevance for the development of AD Neurology. 58 (12), 1791-800 (2002).

55. Braak, H., Alafuzoff, I., Arzberger, T., Kretzschmar, H., Del Tredici, K. Staging of Alzheimer disease-associated neurofibrillary pathology using paraffin sections and immunocytochemistry. Acta Neuropathologica. 112 (4), 389-404 (2006).

56. Mirra, S.S. et al. The Consortium to Establish a Registry for Alzheimer's Disease (CERAD): Part II. Standardization of the neuropathologic assessment of Alzheimer's disease. Neurology. 41 (4), 479-479 (1991).

57. Finger, E.C. Frontotemporal Dementias. CONTINUUM: Lifelong Learning in Neurology. 22 (2, Dementia), $464-489$ (2016).

58. Kovacs, G.G. Neuropathology of Neurodegenerative Diseases. Cambridge University Press. Cambridge. (2014).

59. Kovacs, G.G. et al. Neuropathology of the hippocampus in FTLD-Tau with Pick bodies: a study of the BrainNet Europe Consortium. Neuropathology and applied neurobiology. 39 (2), 166-78 (2013).

60. McKeith, I.G. et al. Diagnosis and management of dementia with Lewy bodies: third report of the DLB Consortium. Neurology. 65, 1863-1872 (2005). 
61. Beach, T.G. et al. Unified staging system for Lewy body disorders: correlation with nigrostriatal degeneration, cognitive impairment and motor dysfunction. Acta neuropathologica. 117 (6), 613-34 (2009).

62. McKeith, I.G. et al. Diagnosis and management of dementia with Lewy bodies. Neurology. 89 (1), 88-100 (2017).

63. Trojanowski, J.Q., Revesz, T., Neuropathology Working Group on MSA Proposed neuropathological criteria for the post mortem diagnosis of multiple system atrophy. Neuropathology and applied neurobiology. 33 (6), 615-20 (2007).

64. Mackenzie, I.R.A. et al. A harmonized classification system for FTLD-TDP pathology. Acta neuropathologica. 122 (1), 111-3 (2011).

65. Nelson, P.T. et al. Limbic-predominant age-related TDP-43 encephalopathy (LATE): consensus working group report. Brain. 142 (6), 15031527 (2019).

66. Josephs, K.A. et al. Staging TDP-43 pathology in Alzheimer's disease. Acta neuropathologica. 127 (3), $441-50$ (2014).

67. Rauramaa, T. et al. Consensus recommendations on pathologic changes in the hippocampus: a postmortem multicenter inter-rater study. Journal of neuropathology and experimental neurology. 72 (6), 452-61 (2013).

68. Iacono, D. et al. Same Ages, Same Genes: Same Brains, Same Pathologies?: Dementia Timings, Co-Occurring Brain Pathologies, ApoE Genotypes in Identical and Fraternal Age-matched Twins at Autopsy. Alzheimer Disease \& Associated Disorders. 30 (2), 178-182 (2016).

69. Guaita, A. et al. Influence of socio-demographic features and apolipoprotein E epsilon 4 expression on the prevalence of dementia and cognitive impairment in a population of 70-74-year olds: The InveCe.Ab study. Archives of Gerontology and Geriatrics. 60 (2), 334-343 (2015).

70. Stevens, M. Factors influencing decisions about donation of the brain for research purposes. Age and ageing. 27 (5), 623-9 (1998).

71. Le Bouc, R. et al. Limiting Factors of Brain Donation in Neurodegenerative Diseases: The Example of French Memory Clinics. Journal of Alzheimer's disease: JAD. 49 (4), 1075-83 (2016).

72. Samarasekera, N. et al. Brain banking for neurological disorders. The Lancet. Neurology. 12 (11), 1096-105 (2013).

73. Beach, T.G. et al. The Sun Health Research Institute Brain Donation Program: description and experience, 1987-2007. Cell and tissue banking. 9 (3), 229-45 (2008).

74. Barnes, L.L., Shah, R.C., Aggarwal, N.T., Bennett, D.A., Schneider, J.A. The Minority Aging Research Study: ongoing efforts to obtain brain donation in African Americans without dementia. Current Alzheimer research. 9 (6), 734-45 (2012).

75. de Lange, G.M., Rademaker, M., Boks, M.P., Palmen, S.J.M.C. Brain donation in psychiatry: results of a Dutch prospective donor program among psychiatric cohort participants. BMC psychiatry. 17 (1), 347 (2017).

76. Peuralinna, T. et al. APOE and AßPP Gene Variation in Cortical and Cerebrovascular Amyloid- $\beta$ Pathology and Alzheimer's Disease: A Population-Based Analysis. Journal of Alzheimer's Disease. 26 (2), 377-385 (2011).

77. Kawas, C.H. The oldest old and the 90+ Study. Alzheimer's \& dementia: the journal of the Alzheimer's Association. 4 (1 Suppl 1), S56-9 (2008).

78. Bennett, D.A., Schneider, J.A., Arvanitakis, Z., Wilson, R.S. Overview and findings from the religious orders study. Current Alzheimer research. 9 (6), 628-45, at <http://www.ncbi.nlm.nih.gov/pubmed/22471860> (2012).

79. O'Brien, R.J. et al. Neuropathologic studies of the Baltimore Longitudinal Study of Aging (BLSA). Journal of Alzheimer's disease: JAD. 18 (3), 665-75 (2009)

80. Jonkman, L.E. et al. Normal Aging Brain Collection Amsterdam (NABCA): A comprehensive collection of postmortem high-field imaging neuropathological and morphometric datasets of non-neurological controls. Neurolmage: Clinical. 22, 101698 (2019).

81. Polito, L. et al. High homocysteine and epistasis between MTHFR and APOE: association with cognitive performance in the elderly. Experimental gerontology. 76, 9-16 (2016).

82. Amzica, F., Lopes da Silva, F. Cellular substrates of brain rhythms. Niedermeyer's Electroencephalography is now in its thoroughly updated sixth edition. 33-63 (2009).

83. Vecchio, F. et al. Resting state cortical EEG rhythms in Alzheimer's disease: toward EEG markers for clinical applications: a review. Supplements to Clinical neurophysiology. 62, 223-36 (2013).

84. Gouw, A.A. et al. EEG spectral analysis as a putative early prognostic biomarker in nondemented, amyloid positive subjects. Neurobiology of Aging. 57, 133-142 (2017).

85. Babiloni, C. et al. Abnormalities of cortical neural synchronization mechanisms in patients with dementia due to Alzheimer's and Lewy body diseases: an EEG study. Neurobiology of Aging. 55, 143-158 (2017).

86. Bonanni, L. et al. Quantitative electroencephalogram utility in predicting conversion of mild cognitive impairment to dementia with Lewy bodies. Neurobiology of Aging. 36 (1), 434-445 (2015).

87. Engedal, K. et al. Quantitative EEG Applying the Statistical Recognition Pattern Method: A Useful Tool in Dementia Diagnostic Workup Dementia and Geriatric Cognitive Disorders. 40 (1-2), 1-12 (2015).

88. Caviness, J.N., Beach, T.G., Hentz, J.G., Shill, H.A., Driver-Dunckley, E.D., Adler, C.H. Association Between Pathology and Electroencephalographic Activity in Parkinson's Disease. Clinical EEG and Neuroscience. 49 (5), 321-327 (2018).

89. Goossens, J. et al. EEG Dominant Frequency Peak Differentiates Between Alzheimer's Disease and Frontotemporal Lobar Degeneration. Journal of Alzheimer's Disease. 55 (1), 53-58 (2016).

90. Bordoni, M. et al. From Neuronal Differentiation of iPSCs to 3D Neuro-Organoids: Modelling and Therapy of Neurodegenerative Diseases. International Journal of Molecular Sciences. 19 (12), 3972 (2018).

91. Alafuzoff, I. Minimal neuropathologic diagnosis for brain banking in the normal middle-aged and aged brain and in neurodegenerative disorders. Handbook of clinical neurology. 150, 131-141 (2018).

92. Wharton, S.B. et al. Epidemiological Neuropathology: The MRC Cognitive Function and Aging Study Experience. Journal of Alzheimer's Disease. 25 (2), 359-372 (2011). 